\title{
Maximally Decimated Perfect-Reconstruction FIR Filter Banks with Pairwise Mirror-Image Analysis (and Synthesis) Frequency Responses
}

\author{
TRUONG Q. NGUYEN, STUDENT MEMBER, IEEe, AND P. P. VAIDYANATHAN, MEMBER, IEEE
}

\begin{abstract}
New structures are presented for the perfect-reconstruction QMF bank, based on lossless building blocks. These structures ensure that the frequency responses of the analysis (and synthesis) filters have pairwise symmetry with respect to $\pi / 2$, and require fewer parameters than recently reported structures (also based on lossless building blocks). The design-time based on the new structures is correspondingly much less than the earlier methods which did not incorporate such symmetry.
\end{abstract}

\section{INTRODUCTION}

Q UADRATURE mirror filters (in short, QMF) are used in a number of communication applications such as subband coders for speech signals [1]-[3], and frequency domain speech scramblers [4]. Fig. 1 shows a typical $M$-channel maximally decimated parallel QMF bank where $H_{k}(z)$ and $F_{k}(z), 0 \leq k \leq M-1$, are analysis and synthesis filters, respectively (we consider only finite impulse response (FIR) filters in this paper). The basic purpose and operation of the filters $H_{k}(z), F_{k}(z)$ can be found in a number of references [1]-[3], [7]-[8], [11][14]. Usually, the reconstructed signal $\hat{x}(n)$ suffers from aliasing, amplitude, and phase distortions [5]. It is sometimes desirable to eliminate all these distortions, so that $\hat{x}(n)=c x\left(n-n_{0}\right)$; such a system is called a perfect reconstruction system (PRS).

The theory of perfect reconstruction when $M$ is a power of two is well known [5], [6]. The design method is based on spectral factorization of an FIR halfband filter. The elegant choices of $H_{0}(z), H_{1}(z), F_{0}(z)$, and $F_{1}(z)$ in [5] and [6] cancel aliasing and yield a perfect reconstruction system. Some methods for perfect reconstruction for arbitrary $M$ have been reported recently [7], [11]. The method in [7] has the property that the analysis and synthesis filters have equal lengths, and provide a paraunitary (or lossless) Alias Component (AC) matrix. Such AC matrices can be obtained by generating the analysis filters based on a class of FIR lattice structures with orthogonal

Manuscript received June 10, 1987; revised October 16, 1987. This work was supported in part by the National Science Foundation under Grants DCI 8552579 and MIP 8604456, and in part by Caltech's Programs in Advanced Technology Grant, sponsored by Aerojet General, General Motors, GTE, and TRW. T. Q. Nguyen was an Aerojet Fellow during the period when this work was performed.

The authors are with the Department of Electrical Engineering, California Institute of Technology, Pasadena, CA 91125.

IEEE Log Number 8719425.

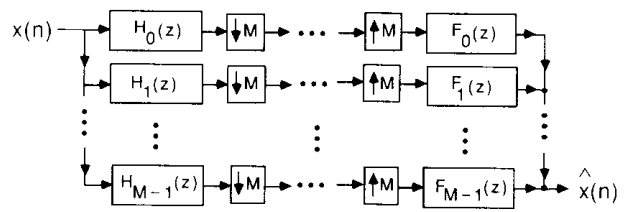

Fig. 1. The $M$-channel maximally decimated parallel QMF bank.

building blocks. With the lattice structures given in [7], the relations among the analysis filters are not explicitly describable, i.e., they are not related in an obvious way. The object of this paper is to describe perfect reconstruction structures for arbitrary $M$ in which the analysis filters $H_{k}(z)$ have pairwise symmetry of frequency responses about $\pi / 2$. To be precise, we consider analysis banks of the form

$$
H_{M-1-k}(z)= \begin{cases}H_{k}(-z), & \text { if } M \text { is odd; } \\ z^{-r} H_{k}\left(-z^{-1}\right), & \text { if } M \text { is even }\end{cases}
$$

for $0 \leq k \leq M-1$ and where $r$ is a positive integer large enough to ensure the causality of $H_{M-1-k}(z)$. An analysis bank satisfying (1) will be called a "pairwise symmetric analysis bank" in this paper. In Section II we recapitulate results on perfect reconstruction structures [7]. Using these conditions with appropriate constraints (1), we derive new perfect reconstruction structures in Sections III and IV, for odd and even $M$, respectively. In each section, we demonstrate theoretical results by design examples. For $M=4$, we compare the filter lengths, the complexity of the analysis bank, and the overall group delay caused by the QMF bank, to the corresponding treestructure based design [5], [18].

The term "quadrature mirror" filters is in fact a misnomer for the analysis/synthesis system of Fig. 1, because the frequency response magnitudes of $H_{k}(z)$ do not in general have symmetry with respect to $\pi / 2$ (which is the "quadrature" of the sampling frequency $2 \pi$ ). One purpose of this paper is to force this symmetry by relating the impulse response coefficients of pairs of filters as in (1). We impose this relation in a way that minimizes the number of parameters that enter the optimization problem [which seeks to minimize the stopband energies of $\left.H_{k}(z)\right]$. 
Even if we do not impose the symmetry conditions of (1), the optimized analysis filters sometimes exhibit such a symmetry, as evidenced from the design example of [7]. However, a priori imposition of such a symmetry condition leads to much faster convergence of the optimization programs, because of elimination of redundant "unknown' parameters. If we wish to impose the condition $\left|H_{k}\left(e^{j \omega}\right)\right|=\left|H_{M-1-k}\left(e^{j \omega}\right)\right|$, then (1) is clearly not the only way to do it. For example, if we arbitrarily replace a zero of $H_{M-1-k}(z)$ with its reciprocal conjugate, then (1) will fail but $\left|H_{k}\left(e^{j \omega}\right)\right|=\left|H_{M-1-k}\left(e^{j \omega}\right)\right|$ will continue to hold. The motivation for choosing the particular relation (1) is the following. For the simple case of $M=$ 2, (1b) is known to hold [5], [6]. With $M=3$, design examples [7] demonstrate that (1a) tends to get satisfied even if this constraint is not explicitly imposed. Moreover, it has been observed that, if the conditions (1a) and (1b) are switched, then further impositions of the losslessness constraint often result in trivial transfer functions. In this paper, we shall therefore restrict our attention only to the type of symmetry in (1).

Notations Used in the Paper: Bold faced italic letters indicate vectors and matrices. Superscript $T$ stands for matrix (or vector) transposition, whereas superscript dagger $(\dagger)$ stands for transposition followed by complex conjugation. Superscript asterisk $(*)$ stands for complex conjugation. The tilde accent on a function $F(z)$ is defined such that, on the unit circle, $\tilde{F}(z)=F^{\dagger}(z)$. In other words, for arbitrary $z, \tilde{F}(z)=F^{T}\left(z^{-1}\right)$. We consider only filters with real coefficients here. A single-input singleoutput digital transfer function $G(z)$ is said to be lossless [8], [15] if it is stable and satisfies $\left|G\left(e^{j \omega}\right)\right|=1$ for all $\omega$. Likewise, an $m$-input $p$-output transfer function matrix $\boldsymbol{T}(z)$ is said to be lossless if it is stable and satisfies the paraunitary property, viz., $\tilde{T}(z) \boldsymbol{T}(z)=I$ for all $z$.

\section{Perfect Reconstruction QMF Bank}

Let the FIR analysis filters $H_{k}(z), k=0, \cdots, M-$ 1 , be written in the form

$$
H_{k}(z)=\sum_{l=0}^{M-1} z^{-l} E_{k, l}\left(z^{M}\right), \quad 0 \leq k \leq M-1
$$

where the above representation is commonly known as polyphase implementation [2], [16] of the $M$-channel structure. Define the $M \times M$ matrix $\boldsymbol{E}(z)=\left[E_{k, l}(z)\right], 0$ $\leq k, l \leq M-1$, then the analysis filters can be expressed as

$$
\left(\begin{array}{c}
H_{0}(z) \\
H_{1}(z) \\
\vdots \\
H_{M-1}(z)
\end{array}\right)=E\left(z^{M}\right)\left(\begin{array}{c}
1 \\
z^{-1} \\
\vdots \\
z^{-(M-1)}
\end{array}\right)
$$

Choose the synthesis filters $F_{k}(z), k=0, \cdots, M-1$ to be

$$
\left(\begin{array}{c}
F_{0}(z) \\
F_{1}(z) \\
\vdots \\
F_{M-1}(z)
\end{array}\right)=z^{-s M} \boldsymbol{E}\left(z^{-M}\right)\left(\begin{array}{c}
z^{-(M-1)} \\
z^{-(M-2)} \\
\vdots \\
1
\end{array}\right)
$$

where $s$ is a positive integer large enough so that $z^{-s} \boldsymbol{E}\left(z^{-1}\right)$ has no positive power of $z$. Fig. 1 now can be redrawn as Fig. 2. Based on standard identities for multirate system [2], Fig. 2 can in turn be redrawn as in Fig. 3 , with ideal channel characteristics assumption. If $\boldsymbol{E}(z)$ in Fig. 3 is lossless, i.e., $\tilde{E}(z) \boldsymbol{E}(z)=\boldsymbol{I}$, then it yields a perfect reconstruction structure [7]. Therefore, as long as $E(z)$ is a lossless transfer matrix and $H_{k}(z), F_{k}(z)$ are defined as in (3) and (4), the structure in Fig. 1 is a perfect reconstruction structure [7]. A simple way to obtain lossless $\boldsymbol{E}(z)$ [8] is to define it as a cascade of lossless systems, i.e., $\boldsymbol{E}(z)=\boldsymbol{K}_{N-1} \boldsymbol{\Lambda}_{N-2}(z) \cdots \boldsymbol{\Lambda}_{0}(z) \boldsymbol{K}_{0}$, where $\boldsymbol{K}_{i}$ are constant unitary matrices, i.e.,

$$
\boldsymbol{K}_{i}^{\dagger} \boldsymbol{K}_{i}=\boldsymbol{I}, \quad 0 \leq i \leq N-1,
$$

and $\boldsymbol{\Lambda}_{i}(z)$ are diagonal matrices with delay elements ( so that $\left.\tilde{\Lambda}_{i}(z) \Lambda_{i}(z)=I\right)$. A typical $\Lambda_{i}(z)$ for $M=4$ could be

$$
\mathbf{\Lambda}_{i}(z)=\left(\begin{array}{ccll}
1 & 0 & 0 & 0 \\
0 & 1 & 0 & 0 \\
0 & 0 & z^{-1} & 0 \\
0 & 0 & 0 & z^{-1}
\end{array}\right)
$$

which is of course an arbitrary example. We consider only QMF banks with real coefficients, so $\boldsymbol{K}_{i}$ are real orthogonal matrices. A simple way to generate an $M \times M$ orthogonal matrix $K_{i}$ is as a sequence of $\left(\begin{array}{c}M \\ 2\end{array}\right)$ planar rotations [7], [9]. The filters $H_{k}(z)$ of the analysis bank are automatically guaranteed [7] to satisfy the condition

$$
\sum_{k=0}^{M-1}\left|H_{k}\left(e^{j \omega}\right)\right|^{2}=1
$$

by the lossless property of $\boldsymbol{E}(z)$. An objective function which represents the stopband energies of the analysis filters is

$$
\phi_{1}=\sum_{k=0}^{M-1} \int_{k \text { th stopband }}\left|H_{k}\left(e^{j \omega}\right)\right|^{2} d \omega .
$$

Since the constraint (7) is enforced by the structure, the passbands of $H_{k}(z)$ are also "good" if their stopbands are good. The optimization of the parameters of $\boldsymbol{K}_{i}$ ( so as to minimize $\phi_{1}$ ) can be done by employing standard gradient algorithms [10]. This is usually time consuming since the objective function is a nonlinear function of many parameters (the parameters being the planar rotation angles mentioned above). Suppose that $H_{k}(z)$ have pairwise symmetry property, i.e., (1), then for the case where 


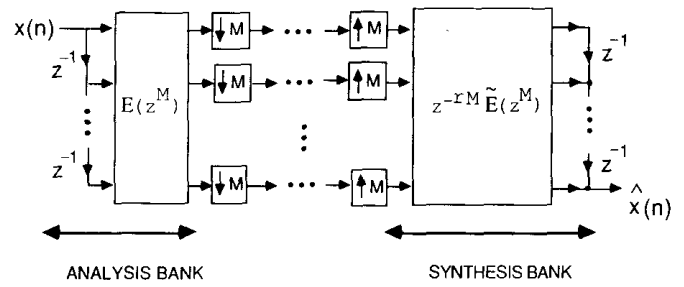

Fig. 2. Polyphase implementation of Fig. 1.

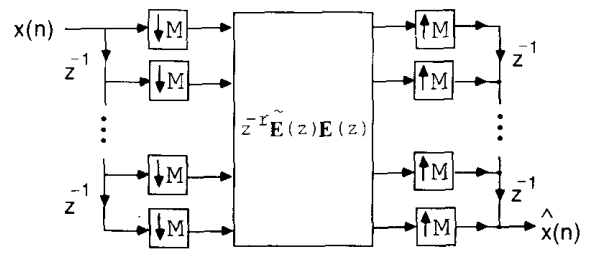

Fig. 3. An equivalent structure of Fig. 2.

$M=3$,

$$
H_{2}(z)=H_{0}(-z), \quad H_{1}(z)=\alpha_{1}\left(z^{2}\right)
$$

for some $\alpha_{1}(z)$. Moreover, it is sufficient to optimize

$$
\begin{aligned}
\phi_{2}= & \int_{(\pi / 3)+\epsilon}^{\pi}\left|H_{0}\left(e^{j \omega}\right)\right|^{2} d \omega \\
& +\int_{0}^{(\pi / 3)-\epsilon}\left|H_{1}\left(e^{j \omega}\right)\right|^{2} d \omega
\end{aligned}
$$

where $\epsilon$ depends on the desired stopband edges. In the next two sections, we modify the structure of Fig. 2 such that the properties described in (1) are structurally enforced. The number of parameters in the resulting structure is approximately half compared to our earlier structure [7]; thus, the design time for this new structure is substantially reduced.

III. Perfect Reconstruction Structures with Pairwise Symmetric Analysis Filters ( $M$ OdD)

Recall that any set of $M$ transfer functions $H_{k}(z), 0 \leq$ $k \leq M-1$ can always be represented as in (2), i.e., as in Fig. 2 where $E(z)$ is an $M \times M$ matrix. If we impose the condition

$$
H_{M-1-k}(z)=H_{k}(-z), \quad 0 \leq k \leq M-1,
$$

then we can write

$$
\left(\begin{array}{l}
H_{k}(z) \\
H_{M-1-k}(z)
\end{array}\right)=\frac{1}{\sqrt{2}}\left(\begin{array}{cc}
1 & 1 \\
1 & -1
\end{array}\right)\left(\begin{array}{l}
\alpha_{k}\left(z^{2}\right) \\
z^{-l_{k}} \alpha_{M-1-k}\left(z^{2}\right)
\end{array}\right)
$$

where $l_{k}$ is some odd integer (with $l_{k}=1$, this representation is always possible). Accordingly, the analysis bank can be redrawn as in Fig. 4 where $L=(M-1) / 2(M$ being odd). In this figure, the $M \times M$ matrix $R$ has the

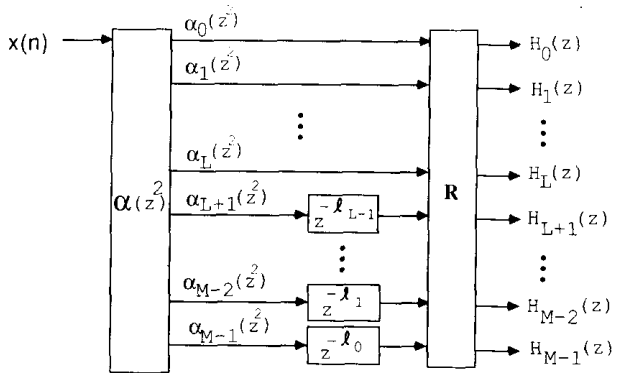

Fig. 4. The analysis bank for $M$ odd.

form

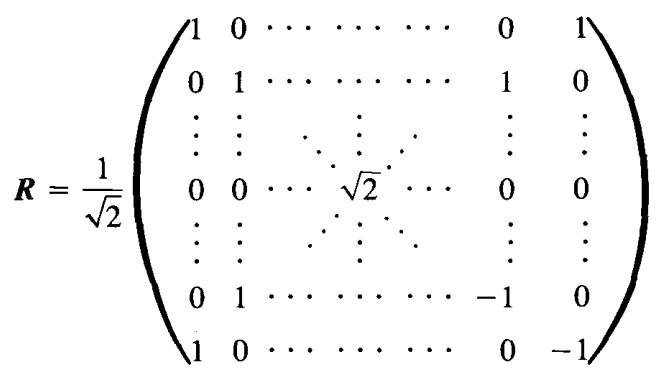

and is orthogonal. By writing $\alpha_{k}(z)$ in the form

$$
\alpha_{k}(z)=\sum_{l=0}^{M-1} z^{-l} E_{k, l}^{\prime}\left(z^{M}\right), \quad 0 \leq k \leq M-1
$$

we can redraw Fig. 4 as in Fig. 5 where $\Gamma^{\prime}(z)$ is an $M \times$ $M$ diagonal matrix of the form,

$$
\Gamma^{\prime}(z)=\left(\begin{array}{ll}
\boldsymbol{I}_{L+1} & \mathbf{0} \\
\mathbf{0} & J_{L}(z)
\end{array}\right)
$$

Here, $J_{L}(z)$ is a diagonal matrix with the diagonal elements $z^{-l_{k}}$. With $l_{k}=1$ for all $k$ ( so that $J_{L}(z)=z^{-1} I_{L}$ ), any set of $M$ transfer functions $H_{k}(z)$ with the constraint (11) can be realized as in Fig. 5 . If we restrict $l_{k}=n_{k} M$ [i.e., integer multiples of $M$ so that $\Gamma^{\prime}(z)=\Gamma\left(z^{M}\right)$ ], we can realize a restricted class of such transfer functions. If $l_{k}$ are so restricted, and if $\boldsymbol{E}^{\prime}(z)$ is lossless, then the complete QMF bank (shown in Fig. 6) is a perfect reconstruction system. This can be seen by drawing it as in Fig. 7 and recognizing that $\tilde{\boldsymbol{E}}^{\prime}\left(z^{2}\right) \tilde{\boldsymbol{\Gamma}}(z) \boldsymbol{R}^{T} \boldsymbol{R} \Gamma(z) \boldsymbol{E}^{\prime}\left(z^{2}\right)=\boldsymbol{I}$. It can be shown that, with the diagonal elements $z^{-l_{k}}$ in $\Gamma^{\prime}(z)$ restricted to be of the form $z^{-n_{k} M}$, where $n_{k}$ are arbitrary integers, losslessness of $\boldsymbol{E}^{\prime}(z)$ in Fig. 5 is equivalent to that of $\boldsymbol{E}(z)$ in Fig. $2 .{ }^{1}$ Furthermore, the synthesis filters $F_{k}(z)$ in Fig. 6 satisfy $F_{k}(z)=$ $z^{-(s M+2 M-2)} \tilde{H}_{k}(z)$, and the synthesis bank in Fig. 6 is equivalent to that of Fig. 2. Consequently, the synthesis filters are pairwise image with respect to $\pi / 2$ if the analysis filters are.

Even though the condition $l_{k}=n_{k} M$ makes it easier to see how to build a perfect reconstruction system [satis-

'See Appendix A for further clarification of this perfect reconstruction property. 


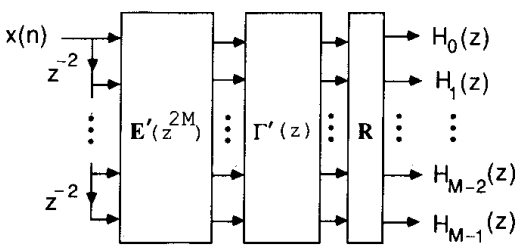

Fig. 5. The $M$-channel analysis bank in which the filter's frequency responses are pairwise image about $\pi / 2$ ( $M$ is odd)

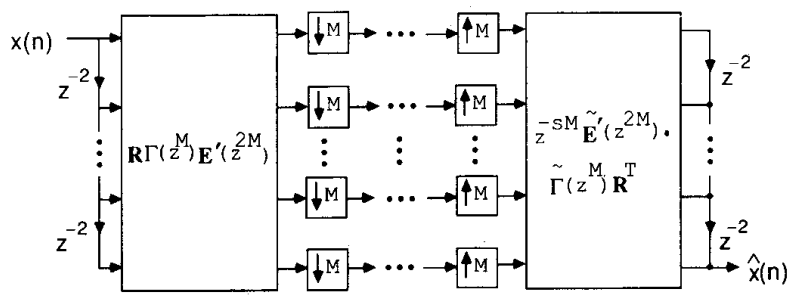

Fig. 6. A QMF bank in which the analysis bank (in Fig. 5) satisfies $\Gamma^{\prime}(z)$ $=\Gamma\left(z^{M}\right)$.

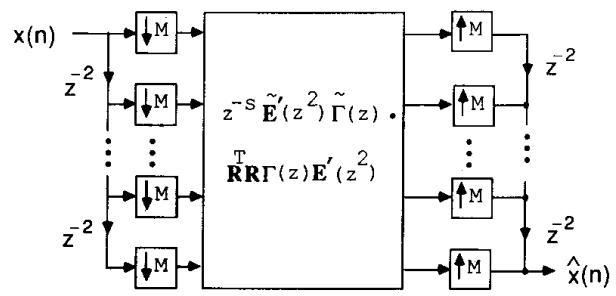

Fig. 7. An equivalent structure for Fig. 6.

fying (11)], this condition is not necessary. As an example, consider an analysis bank as in Fig. 2 with $M=5$ and

$$
\boldsymbol{E}(z)=\left(\begin{array}{rrrrr}
1 & 1 & 0 & 1 & 1 \\
1 & 1 & 0 & -1 & -1 \\
0 & 0 & 2 & 0 & 0 \\
1 & -1 & 0 & 1 & -1 \\
1 & -1 & 0 & -1 & 1
\end{array}\right)
$$

$\boldsymbol{E}(z)$ is orthogonal (and hence lossless) and, moreover, (11) is satisfied. If we now draw the analysis bank as in Fig. 5, we can verify that

$$
\begin{aligned}
& \boldsymbol{E}^{\prime}(z)=\left(\begin{array}{rrrrr}
1 & 0 & 1 & 0 & 0 \\
1 & 0 & -1 & 0 & 0 \\
0 & 2 & 0 & 0 & 0 \\
1 & -1 & 0 & 0 & 0 \\
1 & 1 & 0 & 0 & 0
\end{array}\right), \\
& \Gamma^{\prime}(z)=\left(\begin{array}{ccccc}
1 & 0 & 0 & 0 & 0 \\
0 & 1 & 0 & 0 & 0 \\
0 & 0 & 1 & 0 & 0 \\
0 & 0 & 0 & z^{-1} & 0 \\
0 & 0 & 0 & 0 & z^{-1}
\end{array}\right)
\end{aligned}
$$

so that $\boldsymbol{E}^{\prime}(z)$ is not lossless and $\Gamma^{\prime}(z)$ is not of the form $\Gamma\left(z^{5}\right)$ (i.e., $l_{k}$ not multiples of $M$ ). Yet, it is a perfect reconstruction system satisfying (11)!

In summary, even though Fig. 5 with lossless $\boldsymbol{E}^{\prime}(z)$, orthogonal $\boldsymbol{R}$, and with $\Gamma^{\prime}(z)=\Gamma\left(z^{M}\right)$ (diagonal matrix of delays) leads to a perfect reconstruction system satisfying (11), it does not cover all such systems. We can obtain a relatively more general system by not restricting $\boldsymbol{R}$ to be as in (13). We now proceed to this issue.

Lemma 3.1: Consider the analysis-bank structure of Fig. 5 where $\Gamma^{\prime}(z)$ is of the form

$$
\Gamma^{\prime}(z) \equiv \Gamma\left(z^{M}\right)=\left(\begin{array}{ll}
\boldsymbol{I}_{M-M_{1}} & 0 \\
0 & z^{-M_{M_{1}}}
\end{array}\right)
$$

with $M$ odd and where $M_{1}$ is an integer with $0 \leq M_{1} \leq$ $M-1$. The relation (11) holds if and only if $R$ is of the form

$$
R=\left(\begin{array}{cc}
A & B \\
C & 0 \\
P_{1} A & -P_{1} B
\end{array}\right)
$$

where $A$ is $L \times\left(M-M_{1}\right), B$ is $L \times M_{1}, C$ is $1 \times(M$ $-M_{1}$ ), and $\boldsymbol{P}_{1}$ is the $L \times L$ permutation matrix given by

$$
P_{1}=\left(\begin{array}{cc}
0 & 1 \\
1 & 0
\end{array}\right)
$$

Here, $M_{1}$ is the number of connecting lines between $\boldsymbol{E}^{\prime}\left(z^{2 M}\right)$ and $\boldsymbol{R}$ with delay $z^{-M}$, and $M=2 L+1$. A proof of the above lemma can be found in Appendix B. Notice that $M_{1}$ does not have to be equal to $L$ (which is ( $M$ $-1) / 2$ ), even though this was the natural choice when we derived the structures of Fig. 4 and Fig. 5. Next, by forcing $\boldsymbol{E}^{\prime}(z)$ to be lossless and $\boldsymbol{R}$ to be orthogonal, we can obtain perfect reconstruction. Orthogonality of $\boldsymbol{R}$ implies

$\boldsymbol{R R}^{T}=\left(\begin{array}{cc}\boldsymbol{A} & \boldsymbol{B} \\ \boldsymbol{C} & \mathbf{0} \\ \boldsymbol{P}_{1} \boldsymbol{A} & -\boldsymbol{P}_{1} \boldsymbol{B}\end{array}\right)\left(\begin{array}{ccc}\boldsymbol{A}^{T} & \boldsymbol{C}^{T} & \boldsymbol{A}^{T} \boldsymbol{P}_{1} \\ \boldsymbol{B}^{T} & \mathbf{0}^{T} & -\boldsymbol{B}^{T} \boldsymbol{P}_{1}\end{array}\right)=I$,

which is equivalent to the three following conditions:

$$
\left\{\begin{array}{l}
\boldsymbol{C} C^{T}=1, \\
A A^{T}=B B^{T}=\frac{1}{2} I_{L}, \\
A C^{T}=0 .
\end{array}\right.
$$

Since $\boldsymbol{R}$ is a square matrix, (21) also implies $\boldsymbol{R}^{T} \boldsymbol{R}=\boldsymbol{I}$ which is equivalent to the following conditions:

$$
\left\{\begin{array}{l}
2 A^{T} A+C^{T} C=I_{M-M_{1}}, \\
B^{T} B=\frac{1}{2} I_{M_{1}},
\end{array}\right.
$$

in terms of the submatrices $\boldsymbol{A}, \boldsymbol{B}$, and $\boldsymbol{C}$.

The condition $B B^{T}=\frac{1}{2} I_{L}$ in (22) implies that $M_{1} \geq L$, 
whereas $B^{T} B=\frac{1}{2} I_{M_{1}}$ in (23) implies that $L \geq M_{1}$. In other words, the only choice of $M_{1}$ that is permitted by an orthogonal $R$ of the form (19) is $M_{1}=L . \Gamma^{\prime}(z)$ and $R$ in (18) and (19), respectively, now take simpler forms, i.e.,

$$
\begin{aligned}
\Gamma^{\prime}(z)=\left(\begin{array}{ll}
\boldsymbol{I}_{L+1} & \mathbf{0} \\
\mathbf{0} & z^{-M} \boldsymbol{I}_{L}
\end{array}\right), \\
\text { and } \boldsymbol{R}=\left(\begin{array}{cc}
\boldsymbol{A} & \boldsymbol{B} \\
\boldsymbol{C} & \mathbf{0} \\
\boldsymbol{P}_{1} \boldsymbol{A} & -\boldsymbol{P}_{1} \boldsymbol{B}
\end{array}\right)
\end{aligned}
$$

where $\boldsymbol{A}$ is $L \times(L+1), \boldsymbol{B}$ is $L \times L$, and $\boldsymbol{C}$ is $1 \times(L+$ $1)$. The pairwise symmetric structure of Fig. 5, with $\Gamma^{\prime}(z)$ and $\boldsymbol{R}$ as in (24), is redrawn as Fig. 8. We can simplify the structure in Fig. 8 further by observing that $R$ can be written as

$$
\boldsymbol{R}=\left(\begin{array}{ccc}
\boldsymbol{I}_{L} & \mathbf{0} & \mathbf{0} \\
\mathbf{0} & 1 & \mathbf{0} \\
\mathbf{0} & 0 & \boldsymbol{P}_{1}
\end{array}\right)\left(\begin{array}{ccc}
\boldsymbol{I}_{L} & 0 & \boldsymbol{I}_{L} \\
\mathbf{0} & 1 & \mathbf{0} \\
\boldsymbol{I}_{L} & 0 & -\boldsymbol{I}_{L}
\end{array}\right)\left(\begin{array}{cc}
\boldsymbol{A} & \mathbf{0} \\
\boldsymbol{C} & \mathbf{0} \\
\mathbf{0} & \boldsymbol{B}
\end{array}\right)
$$

Form a square matrix $\boldsymbol{D}$ as follows:

$$
\boldsymbol{D}=\left(\begin{array}{l}
\boldsymbol{A} \\
\boldsymbol{C}
\end{array}\right)_{(L+1) \times(L+1)} .
$$

The orthogonal requirements for $\boldsymbol{A}$ and $\boldsymbol{C}$ in (22) reflect into the following condition on $D$ :

$$
D D^{T}=\left(\begin{array}{ll}
\frac{1}{2} I_{L} & 0 \\
\mathbf{0} & 1
\end{array}\right) .
$$

Such $D$ is easy to construct. For instance, denote the rows of an $(L+1) \times(L+1)$ orthogonal matrix $D^{\prime}$ by $d_{0}^{T}$, $d_{1}^{T}, \cdots, d_{L}^{T}$, then the matrix

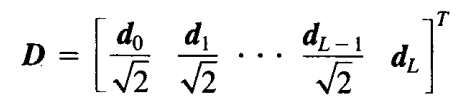

satisfies (27). Using the above orthogonal matrix $D^{\prime}, \boldsymbol{R}$ in (25) is equivalent to

$$
\begin{aligned}
\boldsymbol{R}= & \left(\begin{array}{lll}
\boldsymbol{I}_{L} & 0 & 0 \\
\mathbf{0} & 1 & 0 \\
\mathbf{0} & 0 & \boldsymbol{P}_{1}
\end{array}\right)\left(\begin{array}{llc}
\frac{1}{\sqrt{2}} \boldsymbol{I}_{L} & 0 & \frac{1}{\sqrt{2}} \boldsymbol{I}_{L} \\
\mathbf{0} & 1 & \mathbf{0} \\
\frac{1}{\sqrt{2}} \boldsymbol{I}_{L} & 0 & -\frac{1}{\sqrt{2}} \boldsymbol{I}_{L}
\end{array}\right) \\
& \cdot\left(\begin{array}{ll}
\boldsymbol{D}^{\prime} & \mathbf{0} \\
\mathbf{0} & \sqrt{2} \boldsymbol{B}
\end{array}\right) .
\end{aligned}
$$

We observe from Fig. 8 that $\boldsymbol{D}^{\prime}$ in $\boldsymbol{R}$ can be moved to the left of the delays and can be combined into the general lossless matrix $\boldsymbol{E}^{\prime}(z)$ since the delay lines only affect the last $L$ lines of the structure. Furthermore, as evident from (22), $\hat{\boldsymbol{B}}=\sqrt{2} \boldsymbol{B}$ is an $L \times L$ orthogonal matrix of unity norm. Fig. 8, consequently, can be redrawn as Fig. 9 where $\hat{E}^{\prime}(z)$ is a general $M \times M$ lossless transfer function matrix and $\hat{\boldsymbol{R}}$ is

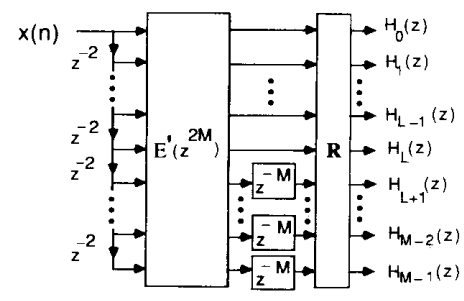

Fig. 8. An equivalent structure for Fig. 5.

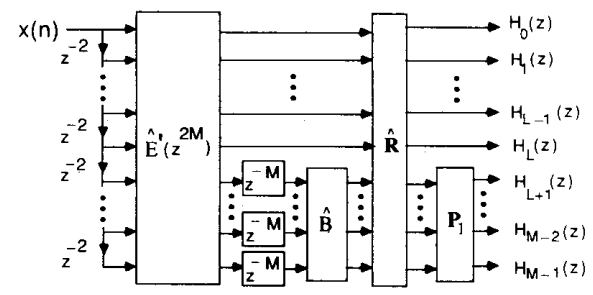

Fig. 9. An equivalent and simplified structure for Fig. 8.

$$
\hat{\boldsymbol{R}}=\left(\begin{array}{ccc}
\frac{1}{\sqrt{2}} I_{L} & 0 & \frac{1}{\sqrt{2}} I_{L} \\
\mathbf{0} & 1 & 0 \\
\frac{1}{\sqrt{2}} I_{L} & 0 & -\frac{1}{\sqrt{2}} I_{L}
\end{array}\right) .
$$

In summary, having chosen $\hat{B}$ to be any $L \times L$ orthogonal matrix of unity norm, the analysis bank in Fig. 9, with $\hat{\boldsymbol{R}}$ defined as in (30), is the analysis bank of a perfect reconstruction structure in which the filters satisfy the pairwise image property.

Example 3.1: Let $M=3$, so that the symmetry requirement (11) on the analysis filters becomes $H_{2}(z)=$ $H_{0}(-z), H_{1}(z)=\alpha_{1}\left(z^{2}\right)$. We also have $L=1$ here. To ensure the lossless property of $\hat{\boldsymbol{E}}^{\prime}(z)$, we choose it to be a cascade of $M \times M$ orthogonal matrices $K_{i}$ and diagonal blocks of delay elements $\boldsymbol{\Lambda}_{i}(z)$. Thus, $\hat{\boldsymbol{E}}^{\prime}(z)=$ $K_{N^{\prime}-1} \Lambda_{N^{\prime}-2}(z) K_{N^{\prime}-2} \Lambda_{N^{\prime}-3}(z) \cdots \Lambda_{0}(z) K_{0}$ where

$$
\begin{aligned}
\boldsymbol{K}_{i}= & \left(\begin{array}{lll}
1 & 0 & 0 \\
0 & \cos \theta_{i, 1} & -\sin \theta_{i, 1} \\
0 & \sin \theta_{i, 1} & \cos \theta_{i, 1}
\end{array}\right) \\
& \cdot\left(\begin{array}{lll}
\cos \theta_{i, 2} & 0 & -\sin \theta_{i, 2} \\
0 & 1 & 0 \\
\sin \theta_{i, 2} & 0 & \cos \theta_{i, 2}
\end{array}\right) \\
& \cdot\left(\begin{array}{lll}
\cos \theta_{i, 3} & -\sin \theta_{i, 3} & 0 \\
\sin \theta_{i, 3} & \cos \theta_{i, 3} & 0 \\
0 & 0 & 1
\end{array}\right), \\
\boldsymbol{\Lambda}_{i}(z)= & \left(\begin{array}{lll}
1 & 0 & 0 \\
0 & 1 & 0 \\
0 & 0 & z^{-1}
\end{array}\right)
\end{aligned}
$$


The terminal lossless block $\hat{\boldsymbol{R}}$ has the form

$$
\hat{\boldsymbol{R}}=\left(\begin{array}{ccc}
\frac{1}{\sqrt{2}} & 0 & \frac{1}{\sqrt{2}} \\
0 & 1 & 0 \\
\frac{1}{\sqrt{2}} & 0 & -\frac{1}{\sqrt{2}}
\end{array}\right)
$$

and $\hat{\boldsymbol{B}}=1$. As a design example, let the number of orthogonal matrices $\boldsymbol{K}_{m}$ be $N^{\prime}=10$, which implies that the order of the analysis filters is 61 . The 30 rotation angles in the lattice structure for $\hat{\boldsymbol{E}}^{\prime}(z)$ were optimized using IMSL subroutines [10] on a computer so as to minimize (10). The resulting frequency response magnitudes for $\epsilon$ $=0.05 \pi$ are shown in Fig. 10 .

Comment on Generality: The main difference of the design technique here, as compared to [7], is that we have structurally imposed the pairwise symmetry conditions (11), so as to cut down the number of planar rotation angles in the optimization. This has led to faster optimization programs, and we have been able to obtain designs with much better stophand attenuation than before. However, we cannot claim that our methods to enforce the symmetry are completely general, as evidenced by the counterexample of (16) and (17). Moreover, the choice of $\boldsymbol{\Lambda}_{i}(z)$ in (31) required for the synthesis of $\hat{\boldsymbol{E}}^{\prime}(z)$ is not general enough. In an earlier conference publication [14], a completely general procedure for synthesizing lossless FIR matrices $\hat{\boldsymbol{E}}^{\prime}(z)$ was outlined. The method in [14] is such that every lossless $\hat{\boldsymbol{E}}^{\prime}(z)$ is covered (and such that none but lossless matrices $\hat{\boldsymbol{E}}^{\prime}(z)$ are covered). Such a general technique can of course be combined with the proposed methods here to obtain further design examples.

\section{Perfect Reconstruction Structures with}

Pairwise Symmetric Analysis Filters ( $M$ Even)

The structure in Fig. 5 is not suitable for even $M$, as it yields analysis filters which are functions of $z^{2}$. When $M$ is even, we define a different requirement on the analysis filters than (11), and still preserve the pairwise symmetry property on the magnitude responses as follows:

$$
H_{M-1-k}(z)=z^{-r} H_{k}\left(-z^{-1}\right) ; \quad 0 \leq k \leq M-1
$$

where $r$ is a positive integer large enough to ensure the causality of $H_{M-1-k}(z)$. For the case of $M=2, H_{1}(z)$ $=z^{-r} H_{0}\left(-z^{-1}\right)$. One recognizes that this is the condition that Smith and Barnwell [5], Mintzer [6], Vaidyanathan and Hoang [18] imposed on their analysis filters for the 2-channel QMF bank. We shall generate this symmetry property by employing the structure of Fig. 11. Here $\boldsymbol{K}_{i}$ are orthogonal matrices. $\boldsymbol{K}_{i}$ and $\boldsymbol{K}_{i+1}$ are separated by a transfer matrix of the form

$$
\left(\begin{array}{ll}
\boldsymbol{I}_{L} & \mathbf{0} \\
\mathbf{0} & z^{-M} \boldsymbol{I}_{L}
\end{array}\right)
$$

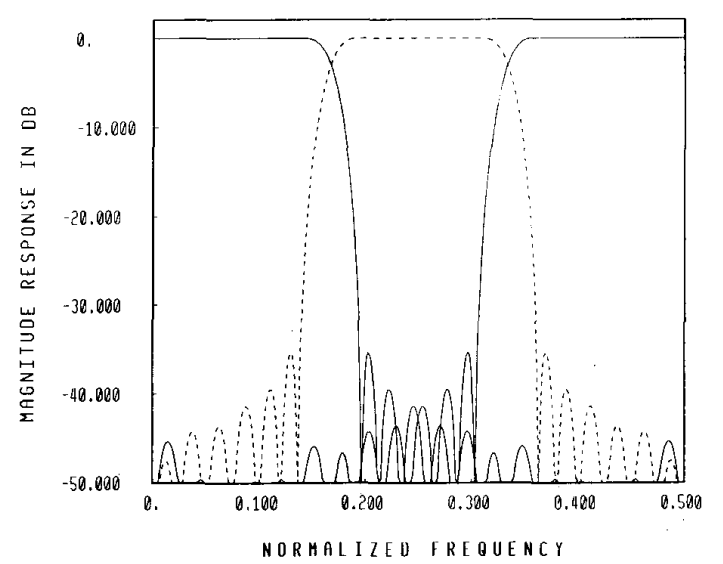

Fig. 10. Magnitude response plots for the optimized analysis filters (Example 3.1).

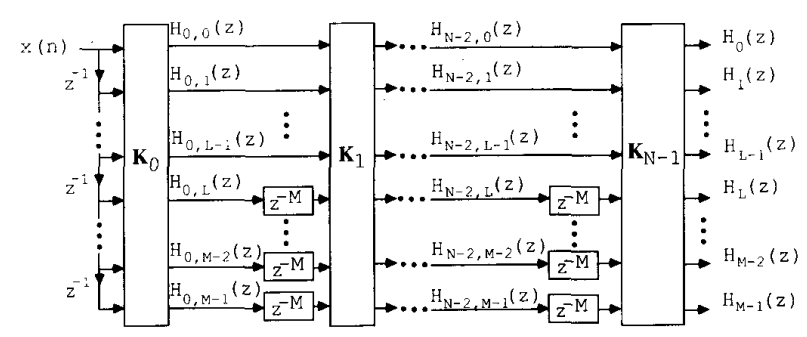

Fig. 11. The $M$-channel analysis bank in which the filter's frequency responses are pairwise image about $\pi / 2$ ( $M$ is even).

where $L=M / 2$. We would like to find $K_{i}$ such that the pairwise symmetry property propagates throughout the structure, i.e., (33) is enforced at every stage of the structure, so that the analysis filters $H_{k}(z)$ also inherit this property. In other words, let $H_{m, k}(z)$ denote the transfer function from the input $x(n)$ to the $k$ th output terminal of $K_{m}$ [Fig. 11 denotes several examples of $H_{m, k}(z)$ ], and suppose that $H_{m, k}(z)$ satisfy (33), i.e. (Fig. 12),

$$
H_{m, M-1-k}(z)=z^{-((m+1) M-1)} H_{m, k}\left(-z^{-1}\right) .
$$

The idea is to find $\boldsymbol{K}_{m+1}$ such that the same property is conserved at the $(m+1)$ th stage, i.e.,

$$
H_{m+1, M-1-k}(z)=z^{-((m+2) M-1)} H_{m+1, k}\left(-z^{-1}\right) .
$$

The only matrix $\boldsymbol{K}_{m+1}$ for which (35) holds for any set of $H_{m, k}(z)$ satisfying (34) is (see Appendix C for proof)

$$
\boldsymbol{K}_{m+1}=\left(\begin{array}{cc}
\boldsymbol{J}_{m+1,1} & \boldsymbol{J}_{m+1,2} \\
-\boldsymbol{P}_{1} \boldsymbol{J}_{m+1,2} \boldsymbol{P}_{1} & \boldsymbol{P}_{1} \boldsymbol{J}_{m+1,1} \boldsymbol{P}_{1}
\end{array}\right)
$$

where $\boldsymbol{P}_{1}$ is defined in (20). Here $\boldsymbol{J}_{m+1,1}$ and $\boldsymbol{J}_{m+1,2}$ are arbitrary $L \times L$ matrices. We observe that using (36) for $K_{0}$ does not yield a set of transfer functions $H_{0, k}(z)$ that satisfy the pairwise symmetric property since the delay chain $\left(1 z^{-1} \cdots z^{-(M-1)}\right)^{T}$ does not satisfy the pairwise symmetry condition in (33). Hence, we need to initiate the induction process by looking for an orthogonal 


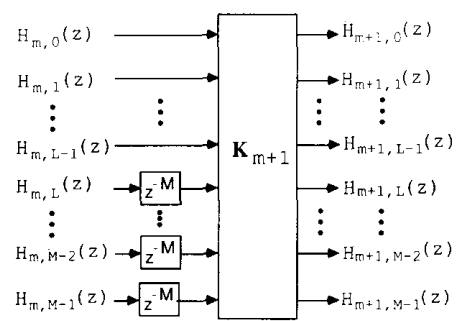

Fig. 12. One stage of the analysis bank in Fig. 11.

matrix $K_{0}$ such that $H_{0, k}(z)$ satisfies (33), for $0 \leq k \leq M$ -1 . Notice that

$$
\left(\begin{array}{l}
H_{0,0}(z) \\
H_{0,1}(z) \\
\vdots \\
H_{0, M-1}(z)
\end{array}\right)=K_{0}\left(\begin{array}{l}
1 \\
z^{-1} \\
\vdots \\
z^{-(M-1)}
\end{array}\right) .
$$

The only $\boldsymbol{K}_{0}$ in (37) for which $H_{0, k}(z)$ satisfies (33) has the form (see Appendix D for proof)

$$
\boldsymbol{K}_{0}=\left(\begin{array}{ll}
\boldsymbol{J}_{0,1} & \boldsymbol{J}_{0,2} \\
\boldsymbol{P}_{1} \boldsymbol{J}_{0,2} \boldsymbol{P}_{3} & \boldsymbol{P}_{1} \boldsymbol{J}_{0,1} \boldsymbol{P}_{2}
\end{array}\right)
$$

where $J_{0,1}, J_{0,2}$ are arbitrary $L \times L$ matrices, $P_{1}$ is as in (20), and

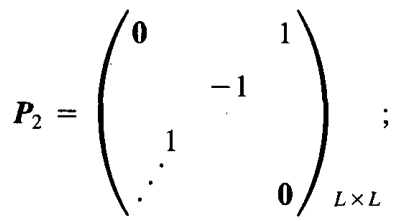

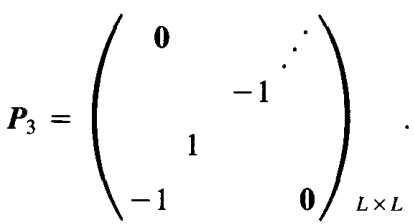

$\boldsymbol{K}_{m+1}$ and $\boldsymbol{K}_{0}$ in (36) and (38) can be rewritten as

$$
\begin{aligned}
\boldsymbol{K}_{m+1} & =\left(\begin{array}{ll}
\boldsymbol{I}_{L} & \mathbf{0} \\
\mathbf{0} & \boldsymbol{P}_{1}
\end{array}\right) \cdot \boldsymbol{T}_{m+1} \cdot\left(\begin{array}{ll}
\boldsymbol{I}_{L} & \mathbf{0} \\
\mathbf{0} & \boldsymbol{P}_{1}
\end{array}\right), \\
\boldsymbol{K}_{0} & =\left(\begin{array}{ll}
\boldsymbol{I}_{L} & \mathbf{0} \\
\mathbf{0} & \boldsymbol{P}_{1}
\end{array}\right) \cdot \boldsymbol{T}_{0} \cdot\left(\begin{array}{ll}
\boldsymbol{I}_{L} & \mathbf{0} \\
\mathbf{0} & \boldsymbol{P}_{2}
\end{array}\right)
\end{aligned}
$$

where

$$
\begin{aligned}
\boldsymbol{T}_{m+1} & =\left(\begin{array}{cc}
\boldsymbol{J}_{m+1,1} & \boldsymbol{J}_{m+1,2} \boldsymbol{P}_{1} \\
-\boldsymbol{J}_{m+1,2} \boldsymbol{P}_{1} & \boldsymbol{J}_{m+1,1}
\end{array}\right), \\
\boldsymbol{T}_{0} & =\left(\begin{array}{ll}
\boldsymbol{J}_{0,1} & -\boldsymbol{J}_{0,2} \boldsymbol{P}_{3} \\
\boldsymbol{J}_{0,2} \boldsymbol{P}_{3} & \boldsymbol{J}_{0,1}
\end{array}\right) .
\end{aligned}
$$

Making use of the identities (40) and (41) in Fig. 11, it can be redrawn as in Fig. 13 where $\boldsymbol{P}_{1}, \boldsymbol{P}_{2}$ are defined as

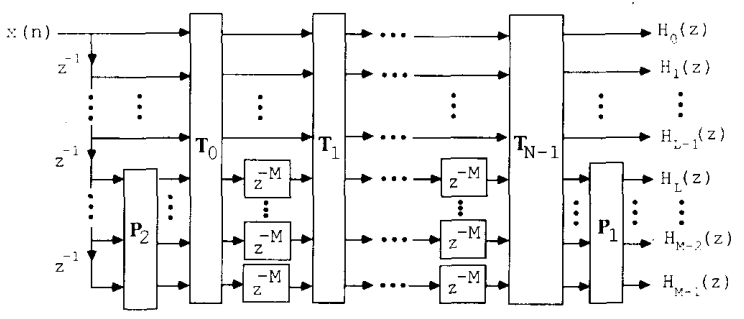

Fig. 13. An equivalent structure of Fig. 11.

in (20) and (39), respectively. Fig. 13 is the analysis bank of a perfect reconstruction structure in which the analysis filters $H_{k}(z)$ and $H_{M-1-k}(z)$ have the pairwise symmetry property if $\boldsymbol{T}_{\boldsymbol{m}+1}$ and $\boldsymbol{T}_{0}$ are orthogonal, i.e., if

$$
T_{m+1}^{T} T_{m+1}=I \quad \text { and } \quad T_{0}^{T} T_{0}=I .
$$

Using the identities

$$
\boldsymbol{P}_{1}^{T}=\boldsymbol{P}_{1}, \quad \boldsymbol{P}_{1}^{2}=\boldsymbol{I}, \quad \text { and } \quad \boldsymbol{P}_{3} \boldsymbol{P}_{2}=-\boldsymbol{I},
$$

(44) is equivalent to

$$
\begin{gathered}
\left\{\begin{array}{l}
\boldsymbol{J}_{m+1,1}^{T} \boldsymbol{J}_{m+1,1}+\boldsymbol{P}_{1} \boldsymbol{J}_{m+1,2}^{T} \boldsymbol{J}_{m+1,2} \boldsymbol{P}_{1}=\boldsymbol{I} \\
\boldsymbol{P}_{1} \boldsymbol{J}_{m+1,1}^{T} \boldsymbol{J}_{m+1,2}=\left(\boldsymbol{P}_{1} \boldsymbol{J}_{m+1,1}^{T} \boldsymbol{J}_{m+1,2}\right)^{T}
\end{array}\right. \\
\text { and }\left\{\begin{array}{l}
\boldsymbol{J}_{0,1}^{T} \boldsymbol{J}_{0,1}+\boldsymbol{P}_{3}^{T} \boldsymbol{J}_{0,2}^{T} \boldsymbol{J}_{0,2} \boldsymbol{P}_{3}=\boldsymbol{I} . \\
\boldsymbol{P}_{2}^{T} \boldsymbol{J}_{0,1}^{T} \boldsymbol{J}_{0,2}=\left(\boldsymbol{P}_{2}^{T} \boldsymbol{J}_{0,1}^{T} \boldsymbol{J}_{0,2}\right)^{T} .
\end{array}\right.
\end{gathered}
$$

If we define

$$
\begin{aligned}
\boldsymbol{U}_{m+1} & =\boldsymbol{J}_{m+1,1}+j \boldsymbol{J}_{m+1,2} \boldsymbol{P}_{1} \quad \text { and } \\
\boldsymbol{U}_{0} & =\boldsymbol{J}_{0,1}+j \boldsymbol{J}_{0,2} \boldsymbol{P}_{3},
\end{aligned}
$$

then it is clear that the unitariness of $\boldsymbol{U}_{m+1}$ and $\boldsymbol{U}_{0}$ implies the orthogonality of $\boldsymbol{T}_{m+1}$ and $\boldsymbol{T}_{0}$, respectively, and vice versa. Consequently, to form orthogonal $M \times M$ matrices $T_{m+1}$ and $T_{0}$ of the specific form (42), (43), we first construct arbitrary $L \times L$ unitary matrices $U_{m+1}$ and $U_{0}$. A general procedure to generate an arbitrary $L \times L$ unitary matrix is described in [9] and requires $L^{2}$ angles. Having formed $\boldsymbol{U}_{m+1}$ and $\boldsymbol{U}_{0}, \boldsymbol{T}_{m+1}$ and $\boldsymbol{T}_{0}$ are constructed as follows:

$$
\begin{aligned}
\boldsymbol{T}_{m+1}=\left(\begin{array}{rr}
\operatorname{Re}\left(\boldsymbol{U}_{m+1}\right) & \operatorname{Im}\left(\boldsymbol{U}_{m+1}\right) \\
-\operatorname{Im}\left(\boldsymbol{U}_{m+1}\right) & \operatorname{Re}\left(\boldsymbol{U}_{m+1}\right)
\end{array}\right), \\
\text { and } \quad \boldsymbol{T}_{0}=\left(\begin{array}{lr}
\operatorname{Re}\left(\boldsymbol{U}_{0}\right) & -\operatorname{Im}\left(\boldsymbol{U}_{0}\right) \\
\operatorname{Im}\left(\boldsymbol{U}_{0}\right) & \operatorname{Re}\left(\boldsymbol{U}_{0}\right)
\end{array}\right) .
\end{aligned}
$$

This procedure guarantees that $\boldsymbol{T}_{m+1}$ and $\boldsymbol{T}_{0}$ are orthogonal and, hence, Fig. 13 is the analysis bank of a perfect reconstruction structure with pairwise symmetric response analysis filters. Compared to our earlier method [7] which requires $\left(\begin{array}{c}2 L \\ 2\end{array}\right)$ rotational angles, the symmetric structure described above requires at most $L^{2}$ angles [9]. Thus, the above structure yields faster optimization algorithms. The relation between $\boldsymbol{T}_{m+1}$ and $\boldsymbol{U}_{m+1}$ leads us 


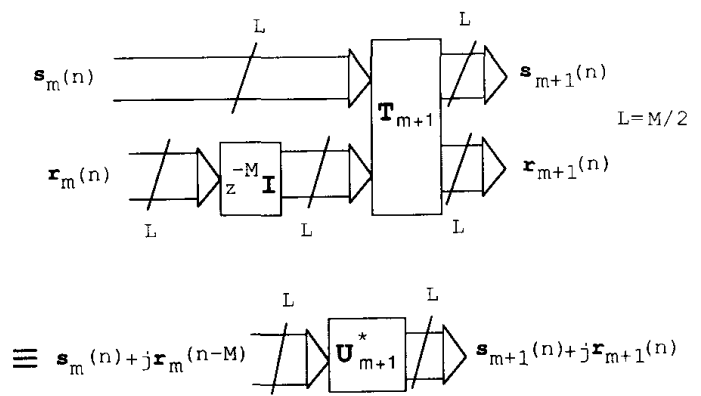

Fig. 14. The "complex domain" interpretation of each stage in Fig. 13.

to the equivalence shown in Fig. 14. Thus, the analysis bank is essentially a cascade of $L \times L$ complex unitary building blocks, with delays inserted into the imaginary paths of the signals. Appropriate adjustments are done at the left and right ends in order to obtain the correct initializations and the correct outputs. The initialization is done by setting

$$
\left(\begin{array}{l}
s_{0}(n) \\
r_{0}(n)
\end{array}\right)=T_{0}\left(\begin{array}{l}
s_{-1}(n) \\
P_{2} r_{-1}(n)
\end{array}\right)
$$

where $s_{-1}(n)=[x(n) x(n-1) \cdots x(n-L+1)]^{T}$ and $r_{-1}(n)=[x(n-L) x(n-L-1) \cdots x(n-2 L$ $+1)]^{T}$ (recall $L=M / 2$ ). The analysis transfer functions are obtained as $\boldsymbol{H}_{0}(z)=\boldsymbol{s}_{N-1}(z) / X(z)$ and $\boldsymbol{H}_{1}(z)=\boldsymbol{P}_{1} \boldsymbol{r}_{N-1}(z) / X(z)$ where $\boldsymbol{H}_{0}(z)=\left[H_{0}(z)\right.$ $\left.H_{1}(z) \cdots H_{L-1}(z)\right]^{T}$ and $H_{1}(z)=\left[H_{L}(z) H_{L+1}(z)\right.$ $\left.\cdots H_{M-1}(z)\right]^{T}$.

Example 4.1: For $M=4$, the analysis filters of a perfect reconstruction structure satisfy the pairwise frequency response image property,

$$
\left\{\begin{array}{l}
H_{3}(z)=z^{-(N M-1)} H_{0}\left(-z^{-1}\right), \\
H_{2}(z)=z^{-(N M-1)} H_{1}\left(-z^{-1}\right)
\end{array}\right.
$$

where $N$ is the number of sections in the structure. Let the unitary matrices $U_{0}$ and $U_{m}$ be

$$
\boldsymbol{U}_{0}=\frac{1}{\sqrt{2}}\left(\begin{array}{cc}
c_{0,1}-j s_{0,1} & c_{0,2}+j s_{0,2} \\
-j\left(c_{0,1}-j s_{0,1}\right) & j\left(c_{0,2}+j s_{0,2}\right)
\end{array}\right),
$$

and

$$
\boldsymbol{U}_{m}=\frac{1}{\sqrt{2}}\left(\begin{array}{cc}
c_{m, 1}+j s_{m, 1} & c_{m, 2}+j s_{m, 2} \\
j\left(c_{m, 1}+j s_{m, 1}\right) & -j\left(c_{m, 2}+j s_{m, 2}\right)
\end{array}\right),
$$

then

$$
\boldsymbol{T}_{0}=\frac{1}{\sqrt{2}}\left(\begin{array}{rrrr}
c_{0,1} & c_{0,2} & s_{0,1} & -s_{0,2} \\
-s_{0,1} & -s_{0,2} & c_{0,1} & -c_{0,2} \\
-s_{0,1} & s_{0,2} & c_{0,1} & c_{0,2} \\
-c_{0,1} & c_{0,2} & -s_{0,1} & -s_{0,2}
\end{array}\right)
$$

and

$$
\boldsymbol{T}_{m}=\frac{1}{\sqrt{2}}\left(\begin{array}{rrrr}
c_{m, 1} & c_{m, 2} & s_{m, 1} & s_{m, 2} \\
-s_{m, 1} & s_{m, 2} & c_{m, 1} & -c_{m, 2} \\
-s_{m, 1} & -s_{m, 2} & c_{m, 1} & c_{m, 2} \\
-c_{m, 1} & c_{m, 2} & -s_{m, 1} & s_{m, 2}
\end{array}\right)
$$

where $c_{m, i}=\cos \left(\theta_{m, i}\right), s_{m, i}=\sin \left(\theta_{m, i}\right)$ and $\theta_{m, i}$ are the planar rotation angles at the $m$ th stage. It can be easily verified that the above $\boldsymbol{T}_{0}$ and $\boldsymbol{T}_{m}$ are orthogonal. The above forms for $\boldsymbol{U}_{0}$ and $\boldsymbol{U}_{m}$ do not represent the most general $2 \times 2$ unitary matrices (which actually require 4 angles to be completely characterized). These forms are meant only to be examples. We choose $N=15$, in this example, so that the length of each (FIR) analysis filter is 60. There are 30 angles $\theta_{m, i} 0 \leq m \leq 14,0 \leq i \leq 1$ in the lattice structure of Fig. 13, and these are optimized using the IMSL subroutine to minimize the following objective function:

$$
\begin{aligned}
\phi= & \int_{(\pi / 4)+\epsilon}^{\pi}\left|H_{0}\left(e^{j \omega}\right)\right|^{2} d \omega+\int_{0}^{(\pi / 4)-\epsilon}\left|H_{1}\left(e^{j \omega}\right)\right|^{2} d \omega \\
& +\int_{(\pi / 2)+\epsilon}^{\pi}\left|H_{1}\left(e^{j \omega}\right)\right|^{2} d \omega
\end{aligned}
$$

for $\epsilon=0.05 \pi$. Note that we do not include the stopband energies of $H_{2}\left(e^{j \omega}\right)$ and $H_{3}\left(e^{j \omega}\right)$ in $\phi$ since they will be small if the stopband energies of their images, $H_{0}\left(e^{j \omega}\right)$ and $H_{1}\left(e^{j \omega}\right)$, are small due to the pairwise symmetry property. Moreover, because of the structural form of Fig. 13, the constraint $\sum_{k=0}^{M-1}\left|H_{k}\left(e^{j \omega}\right)\right|^{2}=1$ automatically holds, hence, the passband errors automatically come out to be small. The magnitude responses of the resulting analysis filters are shown in Fig. 15.

A second procedure to design a four-channel perfectreconstruction system would be to use the tree structure [5] of the form in Fig. 16. Here, $\left[H_{00}(z), H_{01}(z)\right]$ is a two-channel perfect-reconstruction pair, and so is [ $H_{00}^{\prime}(z), H_{01}^{\prime}(z)$ ]. These pairs are designed by spectral factorization of appropriate half-band filters [5]. The overall analysis filters are $H_{0}(z)=H_{00}(z) H_{00}^{\prime}\left(z^{2}\right), H_{1}(z)$ $=H_{00}(z) H_{01}^{\prime}\left(z^{2}\right)$, and so on. In order to obtain the same transition bandwidth $\Delta f$ as in Fig. 15, we should take the transition bandwidth of $H_{00}(z)$ to be $\Delta f$ and that of $H_{00}^{\prime}(z)$ to be $2 \Delta f$. The stopband attenuation seen in Fig. 15 can be obtained with the tree structure if $H_{00}(z)$ and $H_{00}^{\prime}(z)$, designed as in [5], are of lengths 30 and 16 , respectively. Thus, each analysis filter has length $=(29+2 \times 15+$ 1) $=60$. The responses are shown in Fig. 17. It seems to be an interesting coincidence that the analysis filters corresponding to Fig. 15 and Fig. 17 have the same length $(=60)$. It is not clear to us, at this time, as to whether this is a general property of the two methods, for powerof-two $M$. The overall group delay of the QMF system is equal to 59 samples, for both methods.

It is interesting to compare the complexity (in terms of 


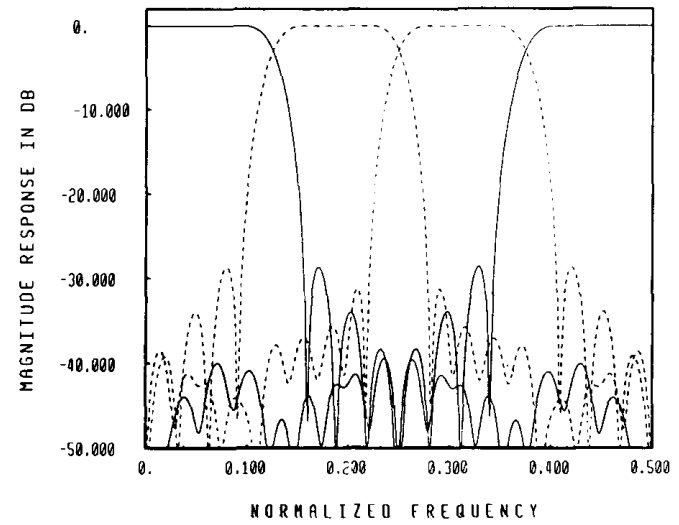

Fig. 15. Magnitude response plots for the optimized analysis filters (Example 4.1).

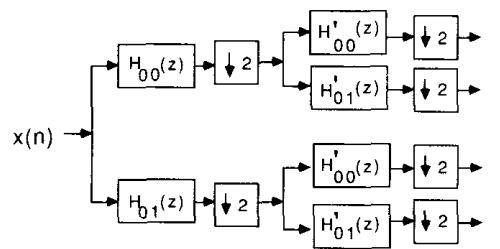

Fig. 16. The $4 \times 4$ tree-structured QMF analysis bank.

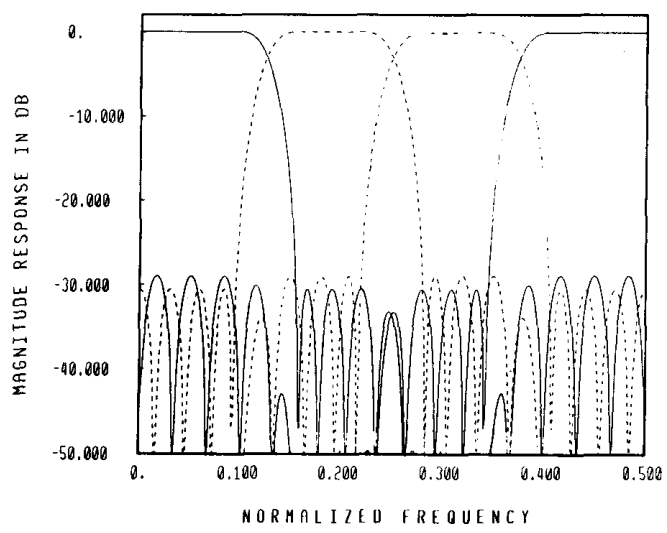

Fig. 17. Magnitude response plots for the analysis filters designed based on the tree structure (Example 4.1).

number of multiplications per unit time (abbreviated $\mathrm{MPU}^{2}$ ) for the two designs. For the tree structured design, we can implement each two-channel branch in Fig. 16 by either direct form [5] or lattice structure [18]. In our implementation, two factors which can potentially contribute to the savings in the number of multiplication are: a) the relation $H_{01}(z)=z^{-29} H_{00}\left(-z^{-1}\right)$, and b) decimation by a factor of two. In a direct-form structure we cannot simultaneously exploit both of these factors because the filter order is necessarily odd (29 in our example). Based on either one of these factors, it is easy to implement the

\footnotetext{
${ }^{2} \mathrm{~A}$ unit of time is defined to be the sampling period of the input sequence $x(n)$ in Fig. 1 .
}

pair $\left[H_{00}(z), H_{01}(z)\right]$ using only $(29+1)$ MPU. Galand and Nussbaumer have proposed a modified direct-form scheme [17] whereby both the above contributing factors can be partially exploited to obtain an implementation with only $3 / 4 \times(29+1)$ MPU. Thus, the pair $\left[H_{00}(z)\right.$, $H_{01}(z)$ ] requires $3 / 4 \times(30)$ multiplications per computed output sample [17]. Similarly, due to the decimation factor, each $\left[H_{00}^{\prime}(z), H_{01}^{\prime}(z)\right]$ pair requires $1 / 2 \times$ $3 / 4 \times(16)=6$ MPU. Hence, in a modified direct-form implementation, the analysis bank designed based on the tree structure requires $3 / 4 \times(30)+3 / 4 \times(16) \approx 35$ MPU.

The lattice structure implementation is based on the lossless property of the polyphase filter matrix $\boldsymbol{E}(z)$ [18]. The denormalized two-channel lattice requires $S / 2$ MPU (see [18]) where $S$ is the length of each analysis filter. The normalized lattice structure, on the other hand, has automatic internal $L_{2}$ scaling property, as the internal building blocks are planar rotation operators. Each such operator is mathematically identical to a single complex multiplication, and can be implemented [20] using 3 real multiplications (and 3 real additions). As a result, the entire normalized analysis bank requires only $3 S / 4 \mathrm{MPU}$ which is exactly the same as the best known [17]. The complexity of the pair $\left[H_{00}(z), H_{01}(z)\right]$, hence, is 23 and 15 MPU for the normalized and denormalized implementations, respectively. Similarly, due to the decimation factor, each $\left[H_{00}^{\prime}(z)\right.$, and $\left.H_{01}^{\prime}(z)\right]$ pair requires 6 and 4 MPU for the normalized and denormalized structures, respectively. Implemented by lattice structure, the total complexity of the analysis bank designed based on tree structure is $23+2 \times 6=35$ MPU and $15+2 \times 4=$ $23 \mathrm{MPU}$ for the normalized and denormalized structures, respectively.

Next, for transfer functions designed based on the proposed pairwise symmetric lattice structure, if we implement $H_{k}(z)$ of length $\hat{S}$ in direct form and if we share the multipliers of $H_{k}(z)$ and $H_{M-1-k}(z)$, a total of $2 \hat{S}=2$ $\times 60=120$ multiplications are involved per computed output sample (see Fig. 30 of [19]). Having shared the multipliers of the pairwise symmetric analysis filters, we cannot, however, take advantage of the decimation factor of 4. A more efficient direct-form implementation is to take advantage of the decimation ratio without sharing the multipliers. This requires a total of $4 \hat{S} / 4=240 / 4=60$ $=\hat{S}$ MPU for the complete analysis bank.

Finally, if we implement the lattice structure of Fig. 13 directly, we can take further advantage of the orthogonal form of $\boldsymbol{T}_{m}$. At the output of each stage, we would have to compute

$$
\left(\begin{array}{l}
y_{0} \\
y_{1} \\
y_{2} \\
y_{3}
\end{array}\right)=\left(\begin{array}{rrrr}
c_{m, 1} & c_{m, 2} & s_{m, 1} & s_{m, 2} \\
-s_{m, 1} & s_{m, 2} & c_{m, 1} & -c_{m, 2} \\
-s_{m, 1} & -s_{m, 2} & c_{m, 1} & c_{m, 2} \\
-c_{m, 1} & c_{m, 2} & -s_{m, 1} & s_{m, 2}
\end{array}\right)\left(\begin{array}{l}
u_{0} \\
u_{1} \\
u_{2} \\
u_{3}
\end{array}\right)
$$


where $u_{i}$ and $y_{i}$ are the inputs and outputs of each block $T_{m}$. Rewrite the above equation as

$$
\begin{aligned}
\left(\begin{array}{l}
y_{0} \\
y_{1}
\end{array}\right)= & \left(\begin{array}{rr}
c_{m, 1} & s_{m, 1} \\
-s_{m, 1} & c_{m, 1}
\end{array}\right)\left(\begin{array}{l}
u_{0} \\
u_{2}
\end{array}\right) \\
& +\left(\begin{array}{rr}
c_{m, 2} & s_{m, 2} \\
s_{m, 2} & -c_{m, 2}
\end{array}\right)\left(\begin{array}{l}
u_{1} \\
u_{3}
\end{array}\right)=\left(\begin{array}{l}
a_{0} \\
a_{1}
\end{array}\right)+\left(\begin{array}{l}
b_{0} \\
b_{1}
\end{array}\right) \\
\left(\begin{array}{l}
y_{2} \\
y_{3}
\end{array}\right)= & \left(\begin{array}{rr}
-s_{m, 1} & c_{m, 1} \\
-c_{m, 1} & -s_{m, 1}
\end{array}\right)\left(\begin{array}{l}
u_{0} \\
u_{2}
\end{array}\right) \\
& +\left(\begin{array}{rr}
-s_{m, 2} & c_{m, 2} \\
c_{m, 2} & s_{m, 2}
\end{array}\right)\left(\begin{array}{l}
u_{1} \\
u_{3}
\end{array}\right)
\end{aligned}
$$

Having computed $\left(\begin{array}{ll}a_{0} & a_{1}\end{array}\right)^{T}$ and $\left(b_{0} b_{1}\right)^{T}$, we have

$$
\left(\begin{array}{l}
y_{2} \\
y_{3}
\end{array}\right)=\left(\begin{array}{c}
a_{1}-b_{1} \\
-a_{0}+b_{0}
\end{array}\right) \text {. }
$$

Since each $2 \times 2$ block in (56) is an orthogonal block, we can compute it using 3 multiplications and 3 additions [20]. Thus, (55) requires 6 multiplications and 10 additions. The complexity of the normalized lattice structure is, therefore, $6 \times$ number of stages $=6 N=6 / 4 \times \hat{S}=$ 90 MPU where $\hat{S}$ is the length of $H_{k}(z)$. Since the decimators can be moved all the way to the left of the building block $T_{0}$ in Fig. 13, the actual number of MPU's is only $\frac{3}{8} \hat{S} \simeq 23$. Notice that the $4 \times 4$ matrix (55) is orthogonal, hence, the lattice structure is automatically $L_{2}$ scaled. It is possible to obtain a more efficient (but denormalized) lattice structure by noting that, if we divide each element in $T_{m}$ by a constant, say $c_{m, 1}$, the responses of $H_{k}(z)$ are unchanged (except for a scale factor.) Thus, (56) and (57) are rewritten as

$$
\begin{aligned}
\left(\begin{array}{l}
y_{0}^{\prime} \\
y_{1}^{\prime}
\end{array}\right)= & \left(\begin{array}{cc}
1 & s_{m, 1}^{\prime} \\
-s_{m, 1}^{\prime} & 1
\end{array}\right)\left(\begin{array}{l}
u_{0} \\
u_{2}
\end{array}\right) \\
& +\left(\begin{array}{cc}
c_{m, 2}^{\prime} & s_{m, 2}^{\prime} \\
s_{m, 2}^{\prime} & -c_{m, 2}^{\prime}
\end{array}\right)\left(\begin{array}{l}
u_{1} \\
u_{3}
\end{array}\right)=\left(\begin{array}{l}
a_{0}^{\prime} \\
a_{1}^{\prime}
\end{array}\right)+\left(\begin{array}{l}
b_{0}^{\prime} \\
b_{1}^{\prime}
\end{array}\right) \\
\left(\begin{array}{l}
y_{2}^{\prime} \\
y_{3}^{\prime}
\end{array}\right)= & \left(\begin{array}{cc}
-s_{m, 1}^{\prime} & 1 \\
-1 & -s_{m, 1}^{\prime}
\end{array}\right)\left(\begin{array}{l}
u_{0} \\
u_{2}
\end{array}\right) \\
& +\left(\begin{array}{rr}
-s_{m, 2}^{\prime} & c_{m, 2}^{\prime} \\
c_{m, 2}^{\prime} & s_{m, 2}^{\prime}
\end{array}\right)\left(\begin{array}{l}
u_{1} \\
u_{3}
\end{array}\right)
\end{aligned}
$$

By noting that ( $\left.\begin{array}{ll}a_{0}^{\prime} & a_{1}^{\prime}\end{array}\right)^{T}$ can be computed by 2 multiplications, each denormalized orthogonal block $T_{m}$ requires only 5 multiplications. Hence, the total complexity for the analysis bank is $5 \hat{S} / 16 \simeq 19 \mathrm{MPU}$.

Table I summarizes the comparison between the new design and the tree-structured design. As evident from it, the complexity of the new pairwise symmetric lattice structure implementation is less than that of the tree-structured implementation. In fact, with a denormalized lattice, the MPU count is the smallest $(=19)$. Besides the complexity advantage, the pairwise symmetric lattice structure can be used for designing filters with arbitrary $M$, which is not necessarily a power of 2 .

\section{Concluding Remarks}

We have described two perfect-reconstruction structures, free of aliasing and distortions of any kind, in which the analysis filter responses are pairwise images with respect to $\pi / 2$. Using this image property to our advantage, the objective function can be simplified so as to include only the stopband energies of about half the number of filters. For both odd and even number of channels, the number of parameters is approximately cut in half and consequently the design time is substantially reduced. Design examples are given to verify the theory.

\section{APPENDIX A}

We shall state and prove two lemmas here.

Lemma A.1: If the analysis filters can be written in two ways:

$$
\begin{aligned}
\left(\begin{array}{c}
H_{0}(z) \\
H_{1}(z) \\
\vdots \\
H_{M-1}(z)
\end{array}\right) & =\boldsymbol{E}\left(z^{M}\right)\left(\begin{array}{l}
1 \\
z^{-1} \\
\vdots \\
z^{-(M-1)}
\end{array}\right) \\
& =G\left(z^{M}\right)\left(\begin{array}{l}
1 \\
z^{-i} \\
\vdots \\
z^{-i(M-1)}
\end{array}\right)
\end{aligned}
$$

where $^{3}(i, M)=1$, then

$$
\tilde{E} E=I \quad \text { if and only if } \tilde{G} G=I .
$$

Lemma A.2: Consider the structure in Fig. 18. If ( $i$, $M)=1$, then this is a perfect reconstruction system.

With $i=2$ and $M$ is odd, the above two lemmas can be applied to Fig. 5 to conclude two features.

1) If $\boldsymbol{E}^{\prime}\left(z^{2 M}\right)$ is lossless and $\boldsymbol{R}$ orthogonal, then $\boldsymbol{R} \Gamma\left(z^{M}\right) \boldsymbol{E}^{\prime}\left(z^{2 M}\right)=\boldsymbol{G}\left(z^{M}\right)$ is lossless, hence, the complete structure in Fig. 6 has perfect reconstruction property by Lemma A.2.

2) Imposing losslessness on $\boldsymbol{E}^{\prime}(z)$ is equivalent to imposing losslessness on $\boldsymbol{E}(z)$.

Proof (Lemma A.1): First consider the $M \times M$ DFT matrix. This is unitary because $W^{\dagger} W=M I$, i.e.,

$$
\sum_{k=0}^{M-1} W^{-l^{\prime} k} W^{l k}= \begin{cases}0, & \text { if } l \neq l^{\prime} \\ M, & \text { if } l=l^{\prime}\end{cases}
$$

\footnotetext{
${ }^{3}$ Here $(i, M)$ denotes the greatest common divisor of $i$ and $M$
} 
TABLE I

Comparison of the Number of Multiplications per Unit Time in the Analysis Bank for Various Four-Channel QMF Bank Implementations. Here "Unit Time” is the Sampling Period Corresponding to the Input $x(n)$ in Fig. 1 . The Normalized Two-Channel Lattice is Assumed to Be IMPLEMENTEd With Three MULTIPLiCations PER SECtion

\begin{tabular}{|c|c|c|c|c|c|c|c|}
\hline & \multicolumn{4}{|c|}{ Tree-Structure Based Design } & \multicolumn{3}{|c|}{ Nontree Lattice-Based Design } \\
\hline & \multicolumn{2}{|c|}{ Direct Form } & \multicolumn{2}{|c|}{ Lattice [18] } & \multirow[b]{2}{*}{ Direct Form } & \multicolumn{2}{|c|}{ New Lattice } \\
\hline & Regular & Galand [17] & Denorm & Norm & & Norm & Denorm \\
\hline $\begin{array}{l}\text { General Expression } \\
\text { for MPU } \\
\text { Number of MPU with }\end{array}$ & $N_{0}+N_{\mathrm{l}}$ & $\frac{3}{4}\left(N_{0}+N_{1}\right)$ & $\frac{1}{2}\left(N_{0}+N_{1}\right)$ & ${ }_{4}^{3}\left(N_{0}+N_{1}\right)$ & $\hat{s}$ & ${ }_{8}^{3} \hat{S}$ & $\frac{5}{16} \hat{S}$ \\
\hline $\begin{aligned} N_{0} & =30 \\
N_{1} & =16 \\
\hat{S} & =60\end{aligned}$ & 46 & 35 & 23 & 35 & 60 & 23 & 19 \\
\hline $\begin{array}{l}\text { Order of } H_{k}(z)= \\
\text { Group Delay }\end{array}$ & 59 & 59 & 59 & 59 & 59 & 59 & 59 \\
\hline
\end{tabular}

- $N_{0}=$ length of the analysis filters in stage 1 (tree structure).

- $N_{1}=$ length of the analysis filters in stage 2 (tree structure)

- $\hat{S}=$ length of analysis filters in the new lattice.

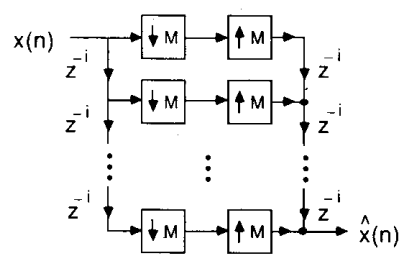

Fig. 18. Pertaining to Appendix A.

where $0 \leq l, l^{\prime} \leq M-1$. Suppose we replace $W$ with $W^{s}$ where $(s, M)=1$, then

$$
\begin{aligned}
\sum_{k=0}^{M-1} W^{-l^{\prime} k s} W^{l k s} & =\sum_{k=0}^{M-1} W^{k s\left(l-l^{\prime}\right)} \\
& = \begin{cases}0, & \text { if } s\left(l-l^{\prime}\right) \neq \text { multiple of } M ; \\
M, & \text { otherwise. }\end{cases}
\end{aligned}
$$

Since $\left|l-l^{\prime}\right|<M$, we see that $s\left(l-l^{\prime}\right)$ is a multiple of $M$ only if $s$ contains a factor of $M$. This cannot happen if $(s, M)=1$. Hence,

$$
\sum_{k=0}^{M-1} W^{k s\left(l-l^{\prime}\right)}= \begin{cases}0, & \text { if } l \neq l^{\prime} \\ M, & l=l^{\prime} .\end{cases}
$$

The modified DFT matrix, denoted $\boldsymbol{W}^{(s)}$, is therefore also unitary. Now (A.1) implies

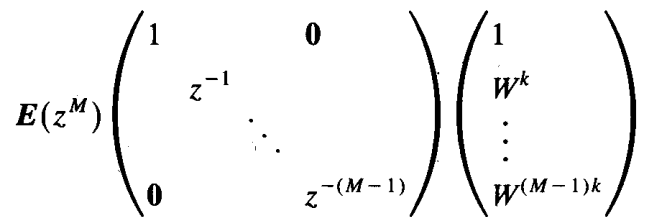

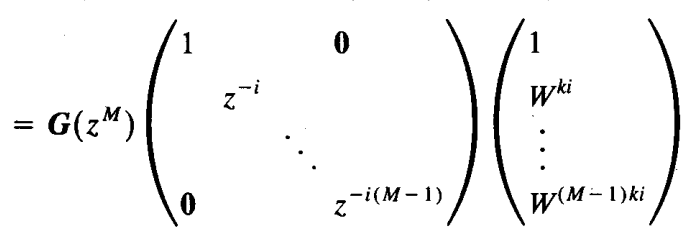

for any $k$. This implies

$$
\boldsymbol{E}\left(z^{M}\right) \boldsymbol{\Lambda}(z) \boldsymbol{W}=\boldsymbol{G}\left(z^{M}\right) \boldsymbol{\Lambda}\left(z^{i}\right) \boldsymbol{W}^{(i)}
$$

where $\boldsymbol{\Lambda}(z)=\operatorname{diag}\left[z^{-k}\right]$. Since $\boldsymbol{\Lambda}(z), \boldsymbol{W}, \boldsymbol{\Lambda}\left(z^{i}\right)$, and $\boldsymbol{W}^{(i)}$ are lossless, we conclude that (A.2) is true as long as $(i, M)=1$.

Proof (Lemma A.2):

$$
\hat{X}(z)=\frac{1}{M} \sum_{k=0}^{M-1} \sum_{l=0}^{M-1} F_{k}(z) H_{k}\left(z W^{l}\right) X\left(z W^{l}\right) \text {. }
$$

In Fig. 18, $H_{k}(z)=z^{-k i}$ and $F_{k}(z)=z^{-(M-1-k) i}$, so (A.6) becomes

$$
\begin{aligned}
\hat{X}(z) & =\frac{1}{M} \sum_{k=0}^{M-1} \sum_{l=0}^{M-1} z^{-(M-1-k) i}\left(z W^{l}\right)^{-k i} X\left(z W^{l}\right) \\
& =\frac{1}{M} \sum_{l=0}^{M-1} X\left(z W^{l}\right) \sum_{k=0}^{M-1} z^{-(M-1-k) i} z^{-k i} W^{-k l i} \\
& =\frac{1}{M} \sum_{l=0}^{M-1} X\left(z W^{l}\right) z^{-(M-1) i} \sum_{k=0}^{M-1} W^{-k l i}
\end{aligned}
$$

Note that $\Sigma_{k=0}^{M-1} W^{-l k i}=0$, unless $l i$ is a multiple of $M$. Since $0 \leq l \leq M-1$ and $(i, M)=1, l$ has to be 0 . Thus, (A.7) simplifies to

$$
\hat{X}(z)=\frac{1}{M} X(z) z^{-(M-1) i} M=z^{-(M-1) i} X(z) .
$$

The structure in Fig. 18 is, therefore, a perfect-reconstruction structure. We will now find the relation between the analysis and synthesis filters of Fig. 6. With $\boldsymbol{G}\left(z^{M}\right)$ $\triangleq \boldsymbol{R} \Gamma\left(z^{M}\right) \boldsymbol{E}^{\prime}\left(z^{2 M}\right)$, the synthesis filters $\boldsymbol{F}_{k}(z)$ in Fig. 6 are given by

$$
\begin{aligned}
& {\left[\begin{array}{llll}
F_{0}(z) & F_{1}(z) & \cdots & F_{M-1}(z)
\end{array}\right]} \\
& =\left[\begin{array}{llll}
z^{-(M-1) i} & z^{-(M-2) i} \cdots z^{-i} & 1
\end{array}\right] \tilde{\boldsymbol{G}}\left(z^{M}\right) z^{-s M}
\end{aligned}
$$


with $i=2$. However,

$$
\begin{aligned}
& {\left[\begin{array}{llll}
\tilde{H}_{0}(z) & \tilde{H}_{1}(z) & \cdots & \tilde{H}_{M-1}(z)
\end{array}\right]} \\
& =\left[\begin{array}{llll}
1 & z^{i} \cdots z^{i(M-1)}
\end{array}\right] \tilde{\boldsymbol{G}}\left(z^{M}\right) \\
& =z^{i(M-1)}\left[z^{-(M-1) i} z^{-(M-2) i} \cdots 1\right] \tilde{\boldsymbol{G}}\left(z^{M}\right) \\
& =z^{i(M-1)}\left[\begin{array}{llll}
F_{0}(z) & F_{1}(z) & \cdots & F_{M-1}(z)
\end{array}\right] z^{s M} .
\end{aligned}
$$

Thus,

$$
F_{k}(z)=z^{-(s M+i M-i)} \tilde{H}_{k}(z) .
$$

If the analysis banks of Fig. 6 and Fig. 2 are related as in (A.1), are the synthesis banks of Fig. 6 and Fig. 2 identical? From (A.1), we have

$$
\begin{aligned}
& {\left[\begin{array}{llll}
1 & z & \cdots & z^{(M-1)}
\end{array}\right] \tilde{\boldsymbol{E}}\left(z^{M}\right)} \\
& \quad=\left[\begin{array}{lll}
1 & z^{i} \cdots z^{i(M-1)}
\end{array}\right] \tilde{\boldsymbol{G}}\left(z^{M}\right)
\end{aligned}
$$

which implies

$$
\begin{aligned}
& z^{(M-1)}\left[z^{-(M-1)} \cdots z^{-1} \quad 1\right] \tilde{E}\left(z^{M}\right) \\
& =z^{i(M-1)}\left[z^{-i(M-1)} \cdots z^{-i} 1\right] \tilde{G}\left(z^{M}\right) .
\end{aligned}
$$

Hence, the synthesis banks in Fig. 6 and Fig. 2 are identical, except for a possible overall delay.

\section{APPENDIX B}

Fig. 19 shows the terminal blocks of the analysis bank for odd $M$ where $M=2 L+1$, and the number of connecting lines with delays $z^{-M}$ are $M_{1} \leq M$. We will derive the necessary and sufficient conditions for $\boldsymbol{R}$ so that $H_{k}(z)$ satisfies the pairwise symmetry property (11). Let

$$
\begin{aligned}
& \boldsymbol{h}^{1}(z)=\left(\begin{array}{c}
H_{0}(z) \\
\vdots \\
H_{L-1}(z)
\end{array}\right), \quad \boldsymbol{h}^{2}(z)=\left(\begin{array}{c}
H_{L+1}(z) \\
\vdots \\
H_{M-1}(z)
\end{array}\right), \\
& \boldsymbol{q}^{1}(z)=\left(\begin{array}{c}
Q_{0}\left(z^{2}\right) \\
\vdots \\
Q_{M-M_{1}-1}\left(z^{2}\right)
\end{array}\right) \\
& \boldsymbol{q}^{2}(z)=\left(\begin{array}{c}
Q_{M-M_{1}}\left(z^{2}\right) \\
\vdots \\
Q_{M-1}\left(z^{2}\right)
\end{array}\right) .
\end{aligned}
$$

Thus, the pairwise symmetry property on $H_{k}(z)$ yields

$$
\boldsymbol{P}_{1} \boldsymbol{h}^{2}(z)=\boldsymbol{h}^{1}(-z)
$$

where $\boldsymbol{P}_{1}$ is defined as in (20). Let $\boldsymbol{R}$ be partitioned into

$$
\boldsymbol{R}=\left(\begin{array}{ll}
\boldsymbol{A} & \boldsymbol{B} \\
\boldsymbol{C} & \boldsymbol{D} \\
\boldsymbol{E} & \boldsymbol{F}
\end{array}\right)_{M \times M}
$$

where $A$ is $L \times\left(M-M_{1}\right), B$ is $L \times M_{1}, C$ is $1 \times(M$ $\left.-M_{1}\right), D$ is $1 \times M_{1}, E$ is $L \times\left(M-M_{1}\right)$, and $F$ is $L \times$

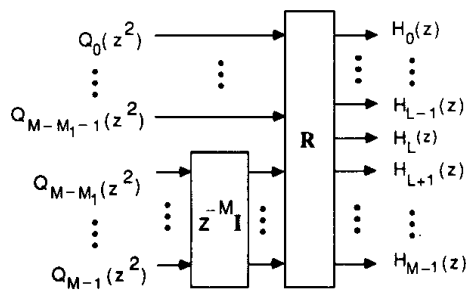

Fig. 19. Pertaining to Appendix B.

$M_{1}$. Thus

$$
\begin{aligned}
\left(\begin{array}{l}
\boldsymbol{h}^{1}(z) \\
H_{L}(z) \\
\boldsymbol{h}^{2}(z)
\end{array}\right) & =\boldsymbol{R} \Gamma\left(z^{M}\right)\left(\begin{array}{c}
\boldsymbol{q}^{1}\left(z^{2}\right) \\
\boldsymbol{q}^{2}\left(z^{2}\right)
\end{array}\right) \\
& =\left(\begin{array}{c}
\boldsymbol{A} \boldsymbol{q}^{1}\left(z^{2}\right)+z^{-M} \boldsymbol{B} \boldsymbol{q}^{2}\left(z^{2}\right) \\
\boldsymbol{C} \boldsymbol{q}^{1}\left(z^{2}\right)+z^{-M} \boldsymbol{D} \boldsymbol{q}^{2}\left(z^{2}\right) \\
\boldsymbol{E} \boldsymbol{q}^{1}\left(z^{2}\right)+z^{-M} \boldsymbol{F} \boldsymbol{q}^{2}\left(z^{2}\right)
\end{array}\right) .
\end{aligned}
$$

Our aim is to find a structural form for $\boldsymbol{R}$ such that, for any set of polynomials $\boldsymbol{q}^{1}(z)$ and $\boldsymbol{q}^{2}(z)$, the vectors $\boldsymbol{h}^{1}(z)$ and $h^{2}(z)$ are related by (A.11). By the pairwise symmetry property, $H_{L}(z)=H_{L}(-z)=$ function of $z^{2}$. Since $M$ is odd, it is clear from (A.13) that $D=0$. Substituting (A.13) into (A.11) and simplifying, we have

$$
\left(\boldsymbol{A}-\boldsymbol{P}_{1} \boldsymbol{E}\right) \boldsymbol{q}^{1}\left(z^{2}\right)=z^{-\boldsymbol{M}}\left(\boldsymbol{P}_{1} \boldsymbol{F}+\boldsymbol{B}\right) \boldsymbol{q}^{2}\left(z^{2}\right) .
$$

Since $M$ is odd, the RHS of (A.14) has only odd powers of $z^{-1}$, whereas the LHS has only even powers. With arbitrary $\boldsymbol{q}^{1}(z)$ and $\boldsymbol{q}^{2}(z)$, this is possible if and only if $A$ $-P_{1} E=0$, and $P_{1} F+B=0$. Thus, $E=P_{1}^{-1} A=P_{1} A$ and $\boldsymbol{F}=-\boldsymbol{P}_{1}^{-1} \boldsymbol{B}=-\boldsymbol{P}_{1} \boldsymbol{B}$ as in (19).

\section{APPENDIX C}

Let

$$
\begin{aligned}
\boldsymbol{h}_{m}^{1}(z) & =\left(\begin{array}{c}
H_{m, 0}(z) \\
\vdots \\
H_{m, L-1}(z)
\end{array}\right) \quad \boldsymbol{h}_{m}^{2}(z)=\left(\begin{array}{c}
H_{m, L}(z) \\
\vdots \\
H_{m, M-1}(z)
\end{array}\right) \\
\boldsymbol{K}_{m+1} & =\left(\begin{array}{ll}
\boldsymbol{A} & \boldsymbol{C} \\
\boldsymbol{B} & D
\end{array}\right)
\end{aligned}
$$

where $\boldsymbol{A}, \boldsymbol{B}, \boldsymbol{C}$, and $\boldsymbol{D}$ have dimension $L \times L$. We will derive the necessary and sufficient condition on $\boldsymbol{K}_{m+1}$ such that (35) holds if (34) holds. In terms of $\boldsymbol{h}_{m}^{1}(z)$ and $\boldsymbol{h}_{m}^{2}(z)$, (34) and (35) are equivalent to

$$
\begin{gathered}
\boldsymbol{P}_{1} \boldsymbol{h}_{m}^{2}(z)=z^{-((m+1) M-1)} \boldsymbol{h}_{m}^{1}\left(-z^{-1}\right), \\
\boldsymbol{P}_{1} \boldsymbol{h}_{m+1}^{2}(z)=z^{-((m+2) M-1)} \boldsymbol{h}_{m+1}^{1}\left(-z^{-1}\right),
\end{gathered}
$$

where $\boldsymbol{P}_{1}$ is as in (20). We would like to find the structural form of $\boldsymbol{K}_{m+1}$ such that (A.16) holds for any set of $\boldsymbol{h}_{m}^{1}(z)$ 
and $\boldsymbol{h}_{m}^{2}(z)$ satisfying (A.15). From Fig. 10

$$
\begin{aligned}
\left(\begin{array}{l}
\boldsymbol{h}_{m+1}^{1}(z) \\
\boldsymbol{h}_{m+1}^{2}(z)
\end{array}\right) & =\left(\begin{array}{ll}
\boldsymbol{A} & \boldsymbol{C} \\
\boldsymbol{B} & \boldsymbol{D}
\end{array}\right)\left(\begin{array}{ll}
\boldsymbol{I}_{L} & \mathbf{0} \\
\mathbf{0} & z^{-M} \boldsymbol{I}_{L}
\end{array}\right)\left(\begin{array}{l}
\boldsymbol{h}_{m}^{1}(z) \\
\boldsymbol{h}_{m}^{2}(z)
\end{array}\right) \\
& =\left(\begin{array}{l}
\boldsymbol{A} \boldsymbol{h}_{m}^{1}(z)+z^{-M} \boldsymbol{C} \boldsymbol{h}_{m}^{2}(z) \\
\boldsymbol{B} \boldsymbol{h}_{m}^{1}(z)+z^{-M} \boldsymbol{D} \boldsymbol{h}_{m}^{2}(z)
\end{array}\right) .
\end{aligned}
$$

Substituting (A.15) into (A.17), we have

$$
\begin{aligned}
\boldsymbol{P}_{1} \boldsymbol{h}_{m+1}^{2}(z)= & -\boldsymbol{P}_{1} \boldsymbol{B} \boldsymbol{P}_{1} \boldsymbol{h}_{m}^{2}\left(-z^{-1}\right) \\
& \cdot z^{-((m+1) M-1)} \\
& +\boldsymbol{P}_{1} D \boldsymbol{h}_{m}^{2}(z) z^{-M} . \quad \text { (A.18) } \\
z^{-((m+2) M-1)} \boldsymbol{h}_{m+1}^{1}\left(-z^{-1}\right)= & \boldsymbol{P}_{1} \boldsymbol{h}_{m}^{2}(z) z^{-M} \\
& +\boldsymbol{C h}_{m}^{2}\left(-z^{-1}\right) z^{-((m+1) M-1)}
\end{aligned}
$$

If (A.16) has to hold, then the right-hand sides of (A.18) and (A.19) should be the same, so that

$$
\begin{gathered}
\left(\boldsymbol{P}_{1} \boldsymbol{B} \boldsymbol{P}_{1}+\boldsymbol{C}\right) \boldsymbol{h}_{m}^{2}\left(-z^{-1}\right) z^{-((m+1) M-1)} \\
=z^{-\boldsymbol{M}}\left(\boldsymbol{P}_{1} \boldsymbol{D}-\boldsymbol{A} \boldsymbol{P}_{1}\right) \boldsymbol{h}_{m}^{2}(z) .
\end{gathered}
$$

For arbitrary causal FIR $\boldsymbol{h}_{m}^{2}(z)$, the function $\boldsymbol{h}_{m}^{2}\left(-z^{-1}\right) z^{-((m+1) M-1)}$ is also causal and FIR, and in general has the form $h_{0}+z^{-1} h_{1}+\cdots$. Since the RHS of (A.20) has power of $z^{-1}$ starting from $z^{-M}$, (A.20) holds for arbitrary $\boldsymbol{h}_{m}^{2}(z)$ if and only if $\boldsymbol{P}_{1} \boldsymbol{B P}+\boldsymbol{C}=\mathbf{0}$, which in turn implies $\boldsymbol{A} \boldsymbol{P}_{1}-\boldsymbol{P}_{1} \boldsymbol{D}=\mathbf{0}$. Simplying these relations, we obtain $B=-\boldsymbol{P}_{1} \boldsymbol{C P}_{1}$ and $D=\boldsymbol{P}_{1} \boldsymbol{A} \boldsymbol{P}_{1}$ which results in the form (36) for $\boldsymbol{K}_{m+1}$.

\section{APPEndix D}

Consider the even $M$ case. Let $\boldsymbol{h}_{-1}^{1}(z)=$ $\left(1 z^{-1} \cdots z^{-(L-1)}\right)^{T}$ and $h_{-1}^{2}=\left(z^{-L} \cdots z^{-(M-1)}\right)^{T}$ where $L=M / 2$. Using the notations in Appendix B with $K_{0}=\left(\begin{array}{cc}A & C \\ B & D\end{array}\right)$, we will derive the necessary and sufficient conditions on $K_{0}$ such that

$$
\begin{aligned}
\left(\begin{array}{l}
\boldsymbol{h}_{0}^{1}(z) \\
\boldsymbol{h}_{0}^{2}(z)
\end{array}\right) & =\left(\begin{array}{ll}
\boldsymbol{A} & \boldsymbol{C} \\
\boldsymbol{B} & \boldsymbol{D}
\end{array}\right)\left(\begin{array}{l}
\boldsymbol{h}_{-1}^{1}(z) \\
\boldsymbol{h}_{-1}^{2}(z)
\end{array}\right) \\
& =\left(\begin{array}{l}
\boldsymbol{A} \boldsymbol{h}_{-1}^{1}(z)+C \boldsymbol{h}_{-1}^{2}(z) \\
B \boldsymbol{h}_{-1}^{1}(z)+D \boldsymbol{h}_{-1}^{2}(z)
\end{array}\right)
\end{aligned}
$$

satisfies the pairwise symmetry property, i.e., $\boldsymbol{P}_{1} h_{0}^{2}(z)=$ $z^{-(M-1)} \boldsymbol{h}_{0}^{1}\left(-z^{-1}\right)$. Accordingly, (A.21) is simplified to

$$
\begin{aligned}
& \boldsymbol{P}_{1} B \boldsymbol{h}_{-1}^{1}(z)+\boldsymbol{P}_{1} D \boldsymbol{h}_{-1}^{2}(z)
\end{aligned}
$$

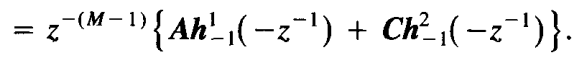

However, $\quad \boldsymbol{h}_{-1}^{1}\left(-z^{-1}\right) z^{-(M-1)}=\boldsymbol{P}_{2} \boldsymbol{h}_{-1}^{2}(z)$, and $\boldsymbol{h}_{-1}^{2}\left(-z^{-1}\right) z^{-(M-1)}=\boldsymbol{P}_{3} \boldsymbol{h}_{-1}^{1}(z)$ where $\boldsymbol{P}_{2}$ and $\boldsymbol{P}_{3}$ are as in (39). Hence, (A.22) simplifies to

$$
\left(\boldsymbol{P}_{1} \boldsymbol{B}-\boldsymbol{C} \boldsymbol{P}_{3}\right) \boldsymbol{h}_{-1}^{\mathbf{1}}(z)=z^{-L}\left(\boldsymbol{A} \boldsymbol{P}_{2}-\boldsymbol{P}_{1} \boldsymbol{D}\right) \boldsymbol{h}_{-1}^{\mathbf{1}}(z)
$$

The matrices $\left(\boldsymbol{P}_{1} \boldsymbol{B}-\boldsymbol{C P}_{3}\right)$ and $\left(\boldsymbol{A} \boldsymbol{P}_{2}-\boldsymbol{P}_{1} \boldsymbol{D}\right)$ are $L \times L$. The left-hand side has the powers $z^{-k}, 0 \leq k \leq L-1$ and the right-hand side contains $z^{-k}, L \leq k \leq M-1$. It can therefore be easily verified that we must have $\boldsymbol{P}_{1} \boldsymbol{B}-$ $C P_{3}=\mathbf{0}, A P_{2}-P_{1} D=0$. This can be rewritten, yielding $B=P_{1} C P_{3}, D=P_{1} A P_{2}$, and thus, $K_{0}$ in (38) is the only form which forces $H_{0, k}(z)$ to have the pairwise symmetry property.

\section{REFERENCES}

[1] D. Esteban and C. Galand, "Application of quadrature mirror filters to split-band voice coding schemes,"' in Proc. IEEE Int. Conf. ASSP, Hartford, CT, May 1977, pp. 191-195.

[2] R. E. Crochiere and L. R. Rabiner, Multirate Digital Signal Processing. Englewood Cliffs, NJ: Prentice-Hall, 1983.

[3] T. P. Barnwell, III, "Subband coder design incorporating recursive quadrature filters and optimum ADPCM coders," IEEE Trans. Acoust., Speech, Signal Processing, vol. ASSP-30, pp. 751-765, Oct. 1982.

[4] R. V. Cox, D. E. Boch, K. B. Bauer, J. D. Johnston, and J. H Snyder, "The analog voice privacy system," in Proc. IEEE Int. Conf. ASSP, Apr. 1986, pp. 341-344.

[5] M. J. Smith and T. P. Barnwell, III, "Exact reconstruction techniques for tree-structured subband coders," IEEE Trans. Acoust., Speech, Signal Processing, vol. ASSP-34, pp. 434-441, June 1986.

[6] F. Mintzer, "Filters for distortion-free two-band multirate filter banks," IEEE Trans. Acoust., Speech, Signal Processing, vol. ASSP33, pp. 626-630, June 1985 .

[7] P. P. Vaidyanathan, "Theory and design of $M$-channel maximally decimated quadrature mirror filters with arbitrary $M$, having perfect reconstruction property,"' IEEE Trans. Acoust., Speech, Signal Processing, vol. ASSP-35, pp. 476-492, Apr. 1987.

[8] P. P. Vaidyanathan and S. K. Mitra, "Low passband sensitivity digital filters: A generalized viewpoint and synthesis procedures," Proc IEEE, pp. 404-423, Apr. 1984.

[9] F. D. Murnaghan, The Unitary and Rotation Groups.、 Washington, DC: Spartan Books, 1962.

[10] The IMSL Library: A set of Fortran subroutines for mathematics and statistics.

[11] M. Vetterli, "A theory of multirate filter banks," IEEE Trans. Acoust., Speech, Signal Processing, vol. ASSP-35, pp. 356-372, Mar. 1987.

[12] T. H. Ramstad, "Analysis/synthesis filter banks with critical sampling," presented at the Int. Conf. Digital Signal Processing, Florence, Italy, Sept. 1984

[13] K. Swaminathan and P. P. Vaidyanathan, "Theory and design of uniform DFT, parallel, quadrature mirror filter banks," IEEE Trans. Circuits and Syst., vol. CAS-33, pp. 1170-1191, Dec. 1986.

[14] P. P. Vaidyanathan, Z. Dog̃anata, and T. Nguyen, "More results on the perfect reconstruction problem in $M$-band parallel QMF banks,", in Proc. IEEE Int. Symp. Circuits and Syst., Philadelphia, PA, May 1987.

[15] V. Belevitch, Classical Network Synthesis. San Francisco, CA: Holden-Day, 1968.

[16] M. G. Bellanger, G. Bonnerot, and M. Coudreuse, "Digital filtering by polyphase networks: Application to sample-rate alternation and filter banks," IEEE Trans. Acoust., Speech, Signal Processing, vol. ASSP-24, pp. 109-114, Apr. 1976.

[17] C. R. Galand and H. J. Nussbaumer, "Quadrature mirror filters with perfect reconstruction and reduced computational complexity," in Proc. IEEE Int. Conf. Acoust., Speech, Signal Processing, Tampa, FL, Apr. 1985, pp. 525-528.

[18] P. P. Vaidyanathan and P.-Q. Hoang, "Lattice structures for optimal design and robust implementation of two-channel perfect-reconstruction QMF banks,"' IEEE Trans. Acoust., Speech, Signal Processing, vol. 36, pp. 81-94, Jan. 1988.

[19] P. P. Vaidyanathan, "Passive cascaded-lattice structures for low-sensitivity FIR filter design, with application to filter banks," IEEE Trans. Circuits and Syst., vol. CAS-33, pp. 1045-1064, Nov. 1986.

[20] R. E. Blahut, Fast Algorithms for Digital Signal Processing. Reading, MA: Addison-Wesley, 1985. 


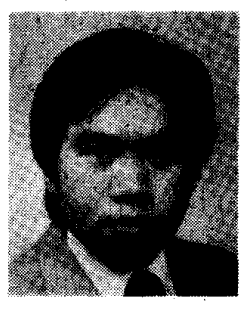

Truong Q. Nguyen (S'86) was born in Saigon, Vietnam, on November 2, 1962. He received the B.S. (hons.) and M.S. degrees from the California Institute of Technology, Pasadena, in electrical engineering in 1985 and 1986 , respectively, and is currently pursuing the Ph.D. degree at the same institution.

During the academic year 1986-1987, he was recipient of a fellowship from Aerojet Dynamics for advanced studies. His main research interests are in digital signal processing, multirate filter bank systems, and filter design.

Mr. Nguyen is a member of Tau Beta Pi and Eta Kappa Nu.

P. P. Vaidyanathan (S'80-M'83), for a photograph and biography, see $\mathrm{p}$. 94 of the March 1988 issue of this TRANSACTIONS. 